\title{
The vacuolar H+ ATPase is a novel therapeutic target for glioblastoma
}

\author{
Andrea Di Cristofori ${ }^{1,2, *}$, Stefano Ferrero ${ }^{3,4, *}$, Irene Bertolini ${ }^{1,3}$, Gabriella Gaudioso ${ }^{1,3}$, \\ Maria Veronica Russo ${ }^{1,3}$, Valeria Berno ${ }^{5}$, Marco Vanini, ${ }^{6}$ Marco Locatelli ${ }^{2}$, Mario \\ Zavanone $^{1,2}$, Paolo Rampini'2, Thomas Vaccari', Manuela Caroli ${ }^{2}$ and Valentina \\ Vaira ${ }^{3,5}$ \\ ${ }^{1}$ Department of Pathophysiology and Organ Transplantation, University of Milan, Milan, Italy \\ 2 Division of Neurosurgery, Fondazione IRCCS Ca' Granda, Ospedale Maggiore Policlinico, Milan, Italy \\ ${ }^{3}$ Division of Pathology, Fondazione IRCCS Ca' Granda, Ospedale Maggiore Policlinico, Milan, Italy \\ ${ }^{4}$ Department of Biomedical, Surgical and Dental Sciences, University of Milan, Milan, Italy \\ ${ }^{5}$ Istituto Nazionale Genetica Molecolare "Romeo ed Enrica Invernizzi", Milan, Italy \\ ${ }^{6}$ Surgical Pathology Unit, St. Anna Hospital, Como, Italy \\ ${ }^{7}$ IFOM - The FIRC Institute of Molecular Oncology, Milan, Italy \\ * These authors have equally contributed to this work \\ Correspondence to: Manuela Caroli, email: man.caroli@tin.it
}

Valentina Vaira, email: vaira@ingm.org

Keywords: glioblastoma, vacuolar H+-ATPase, bafilomycin A 1, cancer stem cells, organotypic tissue cultures Received: January 16, $2015 \quad$ Accepted: May 02, $2015 \quad$ Published: May 22, 2015

This is an open-access article distributed under the terms of the Creative Commons Attribution License, which permits unrestricted use, distribution, and reproduction in any medium, provided the original author and source are credited.

\section{ABSTRACT}

The vacuolar $\mathrm{H}^{+}$ATPase (V-ATPase) is a proton pump responsible for acidification of cellular microenvironments, an activity exploited by tumors to survive, proliferate and resist to therapy. Despite few observations, the role of V-ATPase in human tumorigenesis remains unclear.

We investigated the expression of ATP6V0C, ATP6V0A2, encoding two subunits belonging to the V-ATPase Vo sector and ATP6V1C, ATP6V1G1, ATPT6V1G2, ATP6V1G3, which are part of the $\mathbf{V} 1$ sector, in series of adult gliomas and in cancer stem cellenriched neurospheres isolated from glioblastoma (GBM) patients. ATP6V1G1 expression resulted significantly upregulated in tissues of patients with GBM and correlated with shorter patients' overall survival independent of clinical variables.

ATP6V1G1 knockdown in GBM neurospheres hampered sphere-forming ability, induced cell death, and decreased matrix invasion, a phenotype not observed in GBM monolayer cultures. Treating GBM organotypic cultures or neurospheres with the selective V-ATPase inhibitor bafilomycin A1 reproduced the effects of ATP6V1G1 siRNA and strongly suppressed expression of the stem cell markers Nestin, CD133 and transcription factors SALL2 and POU3F2 in neurospheres.

These data point to ATP6V1G1 as a novel marker of poor prognosis in GBM patients and identify V-ATPase inhibition as an innovative therapeutic strategy for GBM.

\section{INTRODUCTION}

V-ATPase is a ubiquitously expressed and evolutionary conserved multimeric proton pump that acidifies intracellular compartments and the extracellular milieu. V-ATPase is composed of a membrane-spanning V0 sector regulating proton permeability and a V1 sector providing ATPase activity for V0 rotation relative to membranes. Pumping efficiency is modulated by regulated assembly of the two sectors and by coupling of 
ATPase activity with V0 rotation [1]. Multiple roles have been attributed to V-ATPase in physiological conditions, among which regulation of $\mathrm{pH}$ balance, control of neurotransmitter trafficking and degradation of membranebound proteins are the most important $[1,2]$. In addition, recent evidence indicated that $\mathrm{V}$-ATPase acts directly in the endolysosomal compartment to modulate signaling pathways important for development that are dysregulated in cancer, such as Notch, Wnt or mTOR signaling [37]. This evidence, together with the fact that a variety of human tumors display overexpression of V-ATPase subunits, suggests that V-ATPase might play a crucial, albeit largely unexplored, role in supporting tumorigenesis. So far, overexpression of V-ATPase in human tumors has been associated to invasive and chemoresistant phenotypes $[8,9]$ and to increased proliferative activity $[10,11,12]$. Cancer cells are characterized by an abnormal basic intracellular microenvironment, and such $\mathrm{pH}$ imbalance has been proposed to sustain survival by limiting apoptosis, ultimately enhancing cancer proliferation [11]. Thus, V-ATPase could in principle represent a novel target for anti-neoplastic approaches [13-16].

Gliomas are the most frequent primary brain cancers in adults characterized by poor prognosis and limited therapeutic options. Temozolomide, an alkylating agent representing the standard of care, is not curative and overall mortality rates are high $[17,18]$. To date, histologic classification is the most significant prognostic factor in predicting patient survival. Molecular features, including methylation of O6-methylguanine-DNA methyltransferase (MGMT) promoter, isocitrate dehydrogenase 1 or 2 (IDH1/2) gene mutations or $1 \mathrm{p} / 19 \mathrm{q}$ chromosomal loci deletion have recently provided additional information. Nevertheless, limited improvement in prognosis has been achieved, especially in adult patients or for high grades tumors [19, 20].

Dysregulated ion balance in glioma stem cells is emerging as a key factor that could contribute to rapid and aggressive tumor formation and relapse [18]. In line with this evidence, increased expression and/ or activity of V-ATPase, or of other ion transporters is observed in almost all cancers $[11,15,18,21-23]$. Therefore understanding of oncogenic mechanisms depending on selective ion transport could lead to more promising and tailored therapeutic approaches. On these premises, the goal of this study was to determine whether V-ATPase is dysregulated in glioma stem cells and to assess whether V-ATPase targeting could represent an innovative strategy for GBM therapy.

\section{RESULTS}

\section{V-ATPase subunit G1 is up-regulated in human gliomas and in GBM neurospheres}

To get insights on the involvement of V-ATPase dysregulation in human gliomas, we evaluated a panel of V-ATPase subunits belonging to either the V0 (ATP6V0C, ATP6V0A2) or the V1 (ATP6V1C, ATP6V1G1, ATPT6V1G2, ATP6V1G3) sectors (Supplementary Figure 1) using Oncomine, a web-based repository of publicly available series of glioma patients. This analysis showed that ATP6V1G1 and ATP6V0A2 are frequently over-expressed in gliomas compared to non-neoplastic brain tissues without concomitant up-regulation of other subunits (Supplementary Figure 2A, 2B). Available brain cancer series were also investigated for V-ATPase gene amplification. Significant copy number gain for ATP6V1G1 gene located at 9q32 was detected only in recurrent GBMs and not in primary tumors, possibly suggesting that gene amplification might be a later event secondary to gene overexpression (Supplementary Figure 2C).

To confirm these in silico findings in an experimental set-up, we assayed V-ATPase expression at gene or protein expression levels in a small cohort of brain tumors (Gene expression series; Table 1). Among the V-ATPase subunits analyzed, ATP6V1G1 (V-ATPase G1) was the most expressed in human gliomas (Figure 1A and 1B), whereas expression of the V-ATPase G1 paralog ATP6V1G3 was absent in all cases and therefore was excluded from further analysis. Interestingly, ATP6V1G1 expression was also high in commercially available GBM cells (LN229 and T98G) compared to a less aggressive line (SW1088; Supplementary Figure 3A, 3B), and in high-grade human glioma tissues compared to grade II tumors (Figure 1C).

Next, to identify whether V-ATPase subunit expression could be elevated in cancer stem cell enriched samples, we analyzed seven primary neurospheres obtained from GBM patients (Supplementary Table 1) for whom the corresponding tumor tissue and the differentiated primary cell cultures were available (Figure 1D). Elevated gene and protein expression of ATP6V1G1 subunit in neurospheres was detected (Figure $1 \mathrm{E}, 1 \mathrm{~F}$ and Supplementary Figure 4A). Moreover, ATP6V1G1 gene and protein expression significantly decreased when GBM neurospheres were differentiated into adherent cell monolayers (Figure 1G-1I and Supplementary Figure 4B-4D). Finally, V1G1 expression was undetectable in protein extract of grade I brain tumors (Figure 1H, 1I and Supplementary Table 1), whereas it was abundant in rat embryonic hippocampal neuronal cells (E18 RHN; Figure $1 \mathrm{H}, 1 \mathrm{I})$. These data indicate that V-ATPase G1 expression is high in aggressive gliomas and in cancer cell stem cells. 
A

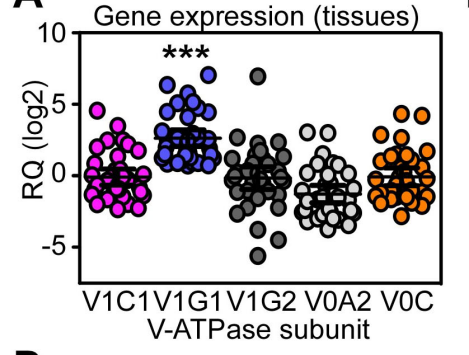

D
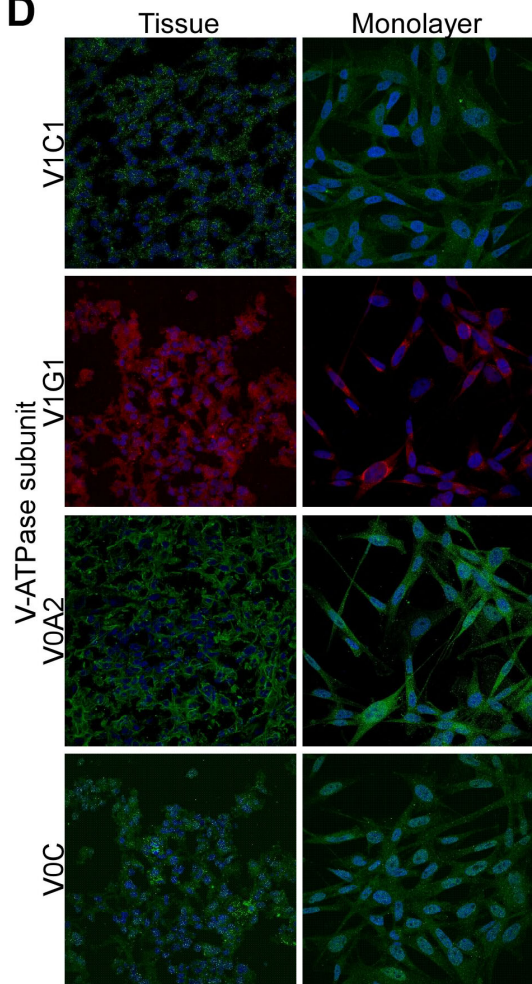

G
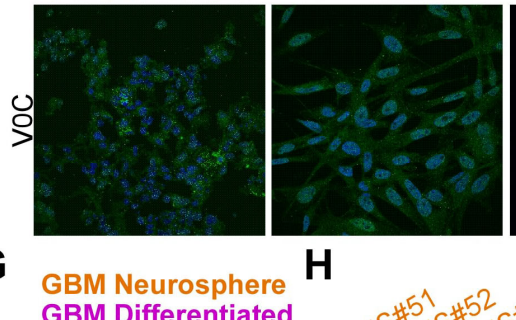

\section{H}

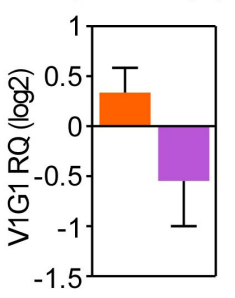

B

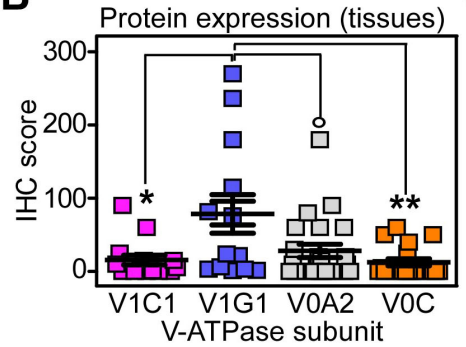

C

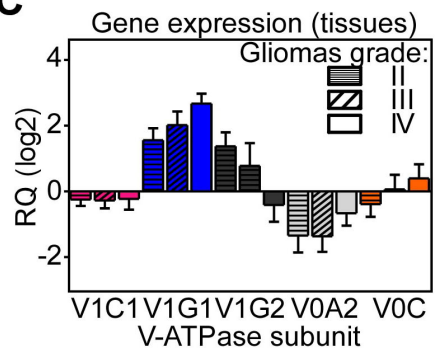

E

E Gene expression

Neurosphere
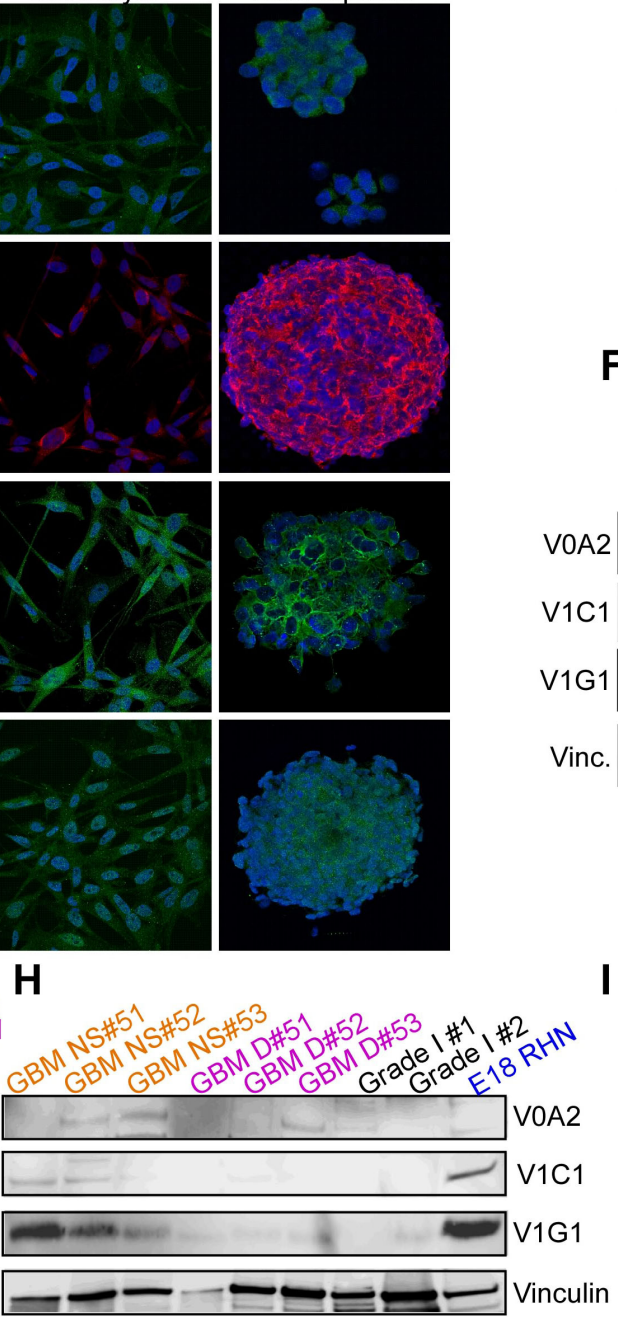
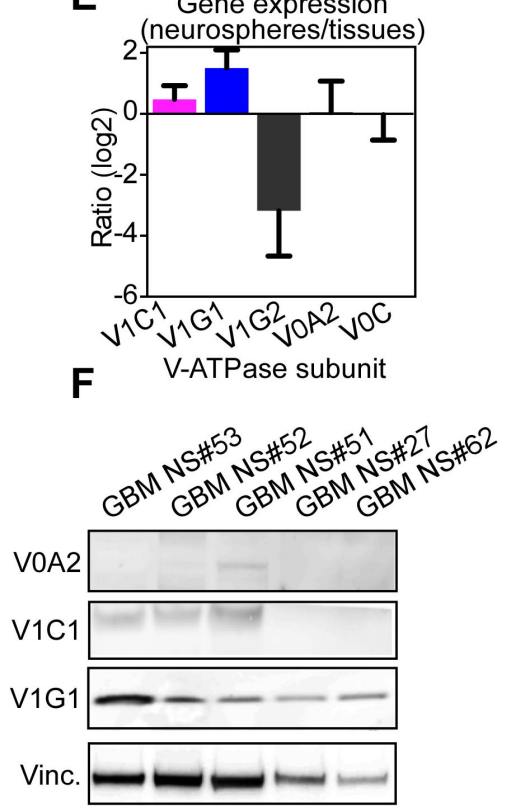

Figure 1: V-ATPase expression in normal brain or gliomas. A., B. The vacuolar proton pump subunits V1C1, V1G1, V1G2, V0A2 and V0C were analyzed by qPCR (A., RQ) or immunohistochemistry (B., IHC score) in a panel of human gliomas $(n=54){ }^{\circ}, p$ $=0.03 ;^{*}, p=0.02{ }^{* *}, p=0.002 ; * * * p<0.0001$ by unpaired $t$ tests. Line, mean with SEM. RQ, mRNA relative quantity. C. V-ATPase subunits expression was investigated in human glioma tissues classified according to WHO grade II, III or IV (GBM). D. Expression of the indicated V-ATPase subunit was analyzed by immunofluorescence in primary glioma tissues and derived neurosphere or differentiated (monolayer) cell cultures from seven individual patients. Representative images are shown. E. Glioblastoma neurospheres obtained from individual patients $(n=7)$ were analyzed for differential gene expression of V-ATPase subunits compared to their original tumor tissues. Bars, mean \pm SD. F. GBM neurospheres obtained from 5 individual patients were analyzed for V-ATPase subunit V0A2, V1C1 and V1G1 protein presence by western blotting. Vinculin (Vinc.) was a loading control. G. Quantification by qRT-PCR of ATP6V1G1 mRNA level in GBM neurospheres or differentiated cultures obtained as in Supplementary Figure 4B ( $n=5$ patients). H. V-ATPase G1 protein expression was analyzed by western blotting in matched GBM neurospheres and differentiated cell monolayers, cultures obtained from Grade I brain tumors (GradeI\#1 and \#2), and embryonic rat hippocampal neural cells (E18 RHN). Vinculin (vinc.) was a loading control. I. Quantification of V-ATPase G1 protein expression obtained as in $\mathrm{H}$ was performed with Image $\mathrm{J}$. ${ }^{*}, p=0.03$ by Wilcoxon signed rank test comparing matched neurosphere and differentiated cultures. 
Table 1: Demographic and clinicopathological characteristics of the glioma patients' series.

\begin{tabular}{|c|c|c|c|c|c|c|c|}
\hline & Grade & Tumor type [abbreviation] & $\mathbf{n}$ & Age $^{1}$ [years] & Gender $[\mathbf{M} / \mathbf{F}]$ & $\begin{array}{l}\text { MGMT methylated } \\
\text { cases }[\mathrm{n}, \%]\end{array}$ & $\begin{array}{l}\text { IDH1 }^{\text {R132H }} \\
{[\mathrm{n}, \%]}\end{array}$ \\
\hline \multirow{6}{*}{ 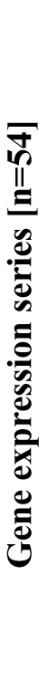 } & \multirow{2}{*}{ 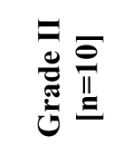 } & Astrocytoma [Astr] & 8 & $43[29-65]$ & $6 \mathrm{M} / 2 \mathrm{~F}$ & $5[62 \%]$ & $2[25 \%]$ \\
\hline & & Oligodendroglioma [OD] & 2 & $25[18-31]$ & $1 \mathrm{M} / 1 \mathrm{~F}$ & $1[50 \%]$ & - \\
\hline & \multirow{3}{*}{ 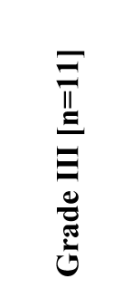 } & Anaplastic Astrocytoma [AA] & 8 & $54[29-78]$ & $5 \mathrm{M} / 3 \mathrm{~F}$ & $3[37.5 \%]$ & $1[12.5 \%]$ \\
\hline & & $\begin{array}{l}\text { Anaplastic } \\
\text { Oligodendroglioma }[\mathrm{AO}]\end{array}$ & 2 & 33 [27-39] & $1 \mathrm{M} / 1 \mathrm{~F}$ & $1[50 \%]$ & $1[50 \%]$ \\
\hline & & $\begin{array}{l}\text { Anaplastic Oligoastrocytoma } \\
{[\mathrm{AOA}]}\end{array}$ & 1 & 49 & $1 \mathrm{M}$ & 1 & - \\
\hline & שี & Glioblastoma [GBM] & 33 & $62[31-82]$ & $20 \mathrm{M} / 13 \mathrm{~F}$ & $16[48.5 \%]$ & - \\
\hline \multirow{5}{*}{ 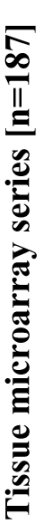 } & \multirow{2}{*}{ 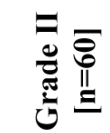 } & Astrocytoma [Astr] & 13 & $41[19-73]$ & $9 \mathrm{M} / 4 \mathrm{~F}$ & $7[53.8 \%]$ & $9[69 \%]$ \\
\hline & & Oligodendroglioma [OD] & 47 & $45[25-72]$ & $28 \mathrm{M} / 19 \mathrm{~F}$ & $39[83 \%]$ & $31[66 \%]$ \\
\hline & \multirow{2}{*}{ 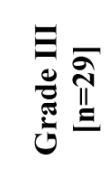 } & Anaplastic Astrocytoma [AA] & 19 & $46[22-78]$ & $11 \mathrm{M} / 8 \mathrm{~F}$ & $10[52.6 \%]$ & $8[42 \%]$ \\
\hline & & $\begin{array}{l}\text { Anaplastic } \\
\text { Oligodendroglioma }[\mathrm{AO}]\end{array}$ & 10 & $52[34-72]$ & $4 \mathrm{M} / 6 \mathrm{~F}$ & $9[90 \%]$ & $8[80 \%]$ \\
\hline & $\frac{2}{0}$ & Glioblastoma [GBM] & 98 & $55[24-78]$ & $59 \mathrm{M} / 39 \mathrm{~F}$ & $48[48.9 \%]$ & $11[11 \%]$ \\
\hline
\end{tabular}

Table 2: Multivariate analysis. Overall survival (OS) was analyzed in GBM patients according to the indicated covariate ${ }^{2}$ using the Cox Proportional-Hazards regression model

\begin{tabular}{|c|c|c|c|c|}
\hline Analysis & Covariate $^{3}$ & $P$ & $\mathbf{H R}^{4}$ & $95 \% \mathrm{CI}^{5}$ \\
\hline \multirow{5}{*}{ OS $[n=70]$} & Age at diagnosis $[\leq 50 \mathrm{y}]$ & 0.007 & 0.5 & $0.2-0.8$ \\
\hline & MGMT methylation & 0.07 & 1.7 & $1-3$ \\
\hline & $\mathrm{KPS}^{6}$ & 0.09 & 0.9 & $0.9-1$ \\
\hline & $\mathrm{IDH} 1^{\mathrm{R} 132 \mathrm{H}}$ & 0.1 & 0.2 & $0.03-1.9$ \\
\hline & V-ATPase G1 score & 0.005 & 3.5 & $1.1-11.4$ \\
\hline
\end{tabular}

${ }^{2}$ Only covariates significant at univariate level were considered (see Supplementary Table 2 for details)

${ }^{3}$ All covariates were categorical. For patients' age, 50 years of age was used as cut-off; for V-ATPaseG1 patients were grouped in low or high expressors according to the protein level established by ROC analysis.

${ }^{4}$ HR, Hazard Ratio

${ }^{5}$ CI, Confidence interval

${ }^{6}$ KPS, Karnofsky Performance Score 


\section{V-ATPase G1 is a marker of poor prognosis for GBM}

We next evaluated whether V-ATPase G1 was significantly enriched in GBM and if was correlated to GBM patients' clinical features or prognosis. Therefore we analyzed V-ATPase G1 immunoreactivity in a larger set of primary gliomas arranged in tissue microarrays (TMAs) derived from 187 patients and in 85 normal brain counterparts (Tissue microarray series; Table 1). Generally, V-ATPase G1 was significantly more abundant in tumor tissues compared to normal cerebral parenchyma (Figure 2A, 2B) and GBM displayed the highest immunoreactivity compared to oligodendroglioma (OD; $p<0.0001)$, anaplastic OD (AO; $p<0.0001)$, astrocytomas (Ast; $p<0.0001$ ) or anaplastic astrocytomas (AA; $p=0.01$ ) (Figure 2B). In normal brain or lowgrade oligodendroglioma samples, V-ATPase G1 was expressed by endothelial cells (arrowheads in Figure 2A). Conversely, in grade III gliomas or GBMs the pump subunit was predominantly localized in tumor cells (Figure 2A). In regards to clinical features of gliomas (Table 1), V-ATPase G1 was more expressed by tumors wild-type for IDH1 enzyme (Figure 2C) whereas it did not correlate to MGMT promoter methylation (Supplementary Figure 5). To investigate whether V-ATPase G1 expression could be used as a prognostic marker, we established a cut-off to sort patients into low- and high-V-ATPase G1 expressing categories using ROC curves (Figure 2D and Supplementary Figure 6). According to this cut-off, corresponding to an immunohistochemistry score of 21.5, GBM patients with high V-ATPase G1 expression had the worst outcome (Figure 2E, 2F and Supplementary Table 2). More importantly, multivariate analysis identified V-ATPase G1 expression as a predictor of shorter overall survival for GBM patients, independent of clinical or molecular features of disease $(p=0.005$ by Cox regression analysis; Table 2).

\section{ATP6V1G1 depletion or V-ATPase inhibition selectively decrease viability of primary GBM neurospheres}

Prompted by the up-regulation of V-ATPase G1 in GBM, we next asked whether knock-down affected proton pump function and cells viability. To address this, we used different GBM models, including LN229 GBM cells, primary GBM monolayer cultures differentiated from neurospheres or patients' derived neurospheres (Supplementary Figure 4). Transient ATP6V1G1 silencing by siRNAs or esiRNA (Figure 3A and Supplementary Figure 7A) effectively decreased protein (Figure 3B) and mRNA levels while expression was not affected by the knock-down of other V-ATPase subunits (Figure 3C and Supplementary Figure 7B). Interestingly, ATP6V1G1 silencing was sufficient to impair V-ATPase-mediated acidification of lysosomes in GBM neurospheres (Figure 3D). In contrast, in differentiated GBM monolayer or LN229 cultures ATP6V1G1 knock-down marginally reduced lysosomal $\mathrm{pH}$ and lysosomal degradation (Supplementary Figure 7C, 7D). Depletion of ATP6V1G1 decreased average neurosphere areas (Figure 3E), induced apoptosis (Figure 3F and Supplementary Figure $8 \mathrm{~A}$ ), and activated effector caspase 3 and 7 (Figure 3G and Supplementary Figure 8B). Cell death triggered by V1G1 depletion did not involve the Bcl2 pro-apoptotic member Bax and did not significantly alter the cell cycle (Supplementary Figure 8C, 8D, respectively). Importantly, V1G1 depletion decreased neurosphere invasion through matrigel (Figure $3 \mathrm{H}$ ) independently of caspases activity (Figure 3H, +ZVAD samples).

In contrast, in adherent glioma cultures or LN229 cells, cell death, caspase $3 / 7$ activity or cell cycle transition was not affected by ATP6V1G1 levels (Supplementary Figure 9A-9F). Cell motility of LN229 cells after ATP6V1G1 knock-down was modestly impaired (Supplementary Figure 9G, 9H), with a reduction of invaded cells of about 30\% (Supplementary Figure 9H), whereas cell invasion was not impaired by V1G1 depletion in differentiated GBM cultures (Supplementary Figure 9I). Finally, we monitored sphere-forming ability of primary GBM cells transfected with a control, an ATP6V1G1directed siRNA or esiRNA over a period of 72 hours. ATP6V1G1 depletion severely restrained formation of neurospheres, compared to control transfected or untreated samples (Figure 4A and Supplementary Movies). As expected [18], GBM neurospheres from different patients displayed heterogeneous clonogenic potential (Figure 4B). We therefore analyzed whether V-ATPase G1 levels were correlated with spheres clonogenicity also in response to ATP6V1G1 gene knockdown. Remarkably, V1G1 was more enriched in GMB patients with higher sphereforming ability (Figure 4B, 4C), and a lower ATP6V1G1 gene expression level (GBM\#27 and 53 samples) was associated to reduced effect of V1G1 knockdown on sphere-forming ability of GBM cultures (Figure 4D).

Spurred by the effects of V-ATPase G1 knock-down on GBM samples, we explored inhibition of V-ATPase activity with the selective inhibitor bafilomycin A1 (BafA1), as a strategy to hamper GBM cell viability. Acute treatment (48 hours) of GBM neurospheres or monolayers with different concentrations of BafA1 $(20 \mathrm{nM}-5 \mu \mathrm{M}$ range) strongly reduced lysosomal acidification (Figure 5A, Supplementary Figure 10A, 10B). This effect was accompanied by a significant reduction of neurospheres area (Figure 5B). Quantification of DNA content in neurospheres treated with BafA1 revealed that toxicity progressively increased with the drug concentration, with approximately $25 \%$ of the cells in sub-G1 phase upon acute treatment with $5 \mu \mathrm{M}$ BafA1 (Figure 5C,5D). As determined by annexin $\mathrm{V}$ staining, apoptosis was activated 
A
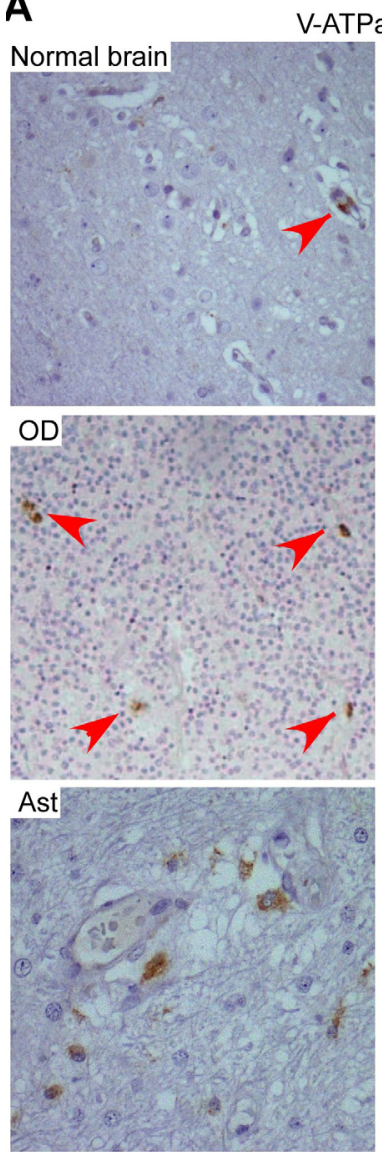

E

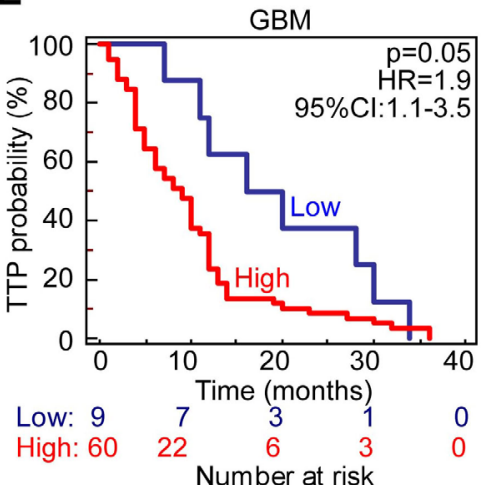

B
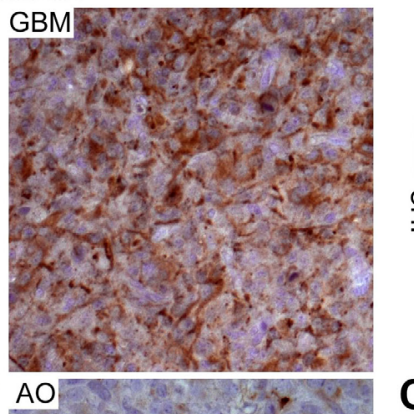
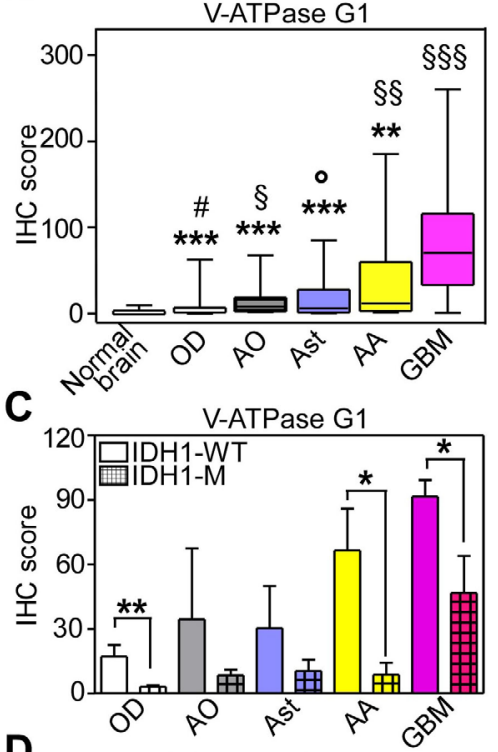

D

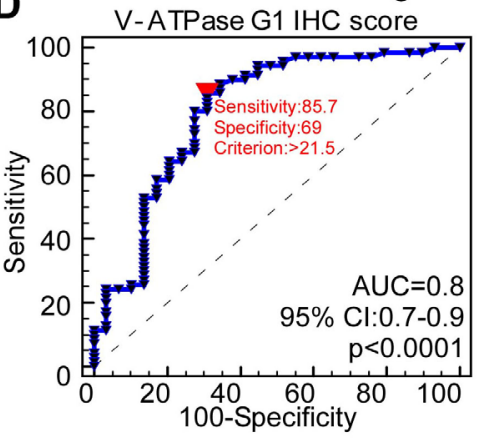

$\mathbf{F}$

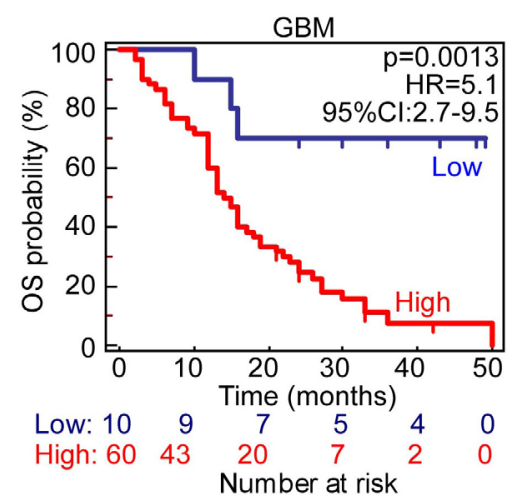

Figure 2: V-ATPase subunit G1 is a novel marker of poor prognosis in GBM patients. A., B. A glioma tissue microarray comprising 85 cores of normal brain parenchyma was analyzed for V-ATPase G1 expression by immunohistochemistry (IHC) A. Photomicrographs representing V-ATPase G1 immunoreactivity in normal brain tissue or in the indicated glioma histotype. Arrowheads indicate brain endothelium. B. V-ATPase G1 IHC score was obtained by multiplying the percentage of positive cells (range 0 - $100 \%$ ) and the staining intensity (range: $0-3$ ). According to this score, normal brain was almost negative (median IHC score 0; range: 0-10). Oligodendrogliomas (OD; median IHC score 1.75; range: 0-62.5), anaplastic OD (AO; median IHC score 6.1; range: 1.5-67.5), astrocytomas (Astr; median IHC score 6.2; range: 0.25-85), anaplastic astrocytomas (AA; median IHC score 11.75; range: 1-185) displayed higher V-ATPase G1 expression compared to normal brain. ${ }^{*}, p=0.0004 ;{ }^{\S}, p=0.047 ;{ }^{\circ}, p=0.038 ; \$ \S, p=0.005 ; \$ \S \$, p<0.0001$ by unpaired $t$-test with Welch's correction, respectively. GBMs scored the highest expression for the subunit G1 (median IHC score 70.25; range: 0.5-260) compared to normal brain $(\S \S \S, p<0.0001)$ and to lower grades gliomas (**, $p=0.012 ; * * *, p<0.001$ by unpaired $t$-test with Welch's correction). C. V-ATPase G1 expression according to R132H mutation of IDH1 gene in different glioma histotypes. Bars, mean \pm SEM. *, $p=0.01 ; * *, p=0.009$ by unpaired $t$-test. IDH1-WT or M, absence or presence of R132H mutation; D. ROC curve was used to identify a cut-off of V-ATPase subunit G1 IHC score (indicated by a red triangle) for patients' categorization into low- or high-expressors. AUC, Area under curve. 95\% CI, 95\% Confidence Interval. E., F. Time to progression (TTP, E) or overall survival (OS, F) end-points were evaluated in GBM patients according to V-ATPase G1 level using the Kaplan-Meier method. P values are from Log-rank test. HR, Hazard Ratio; CI, Confidence Interval. 

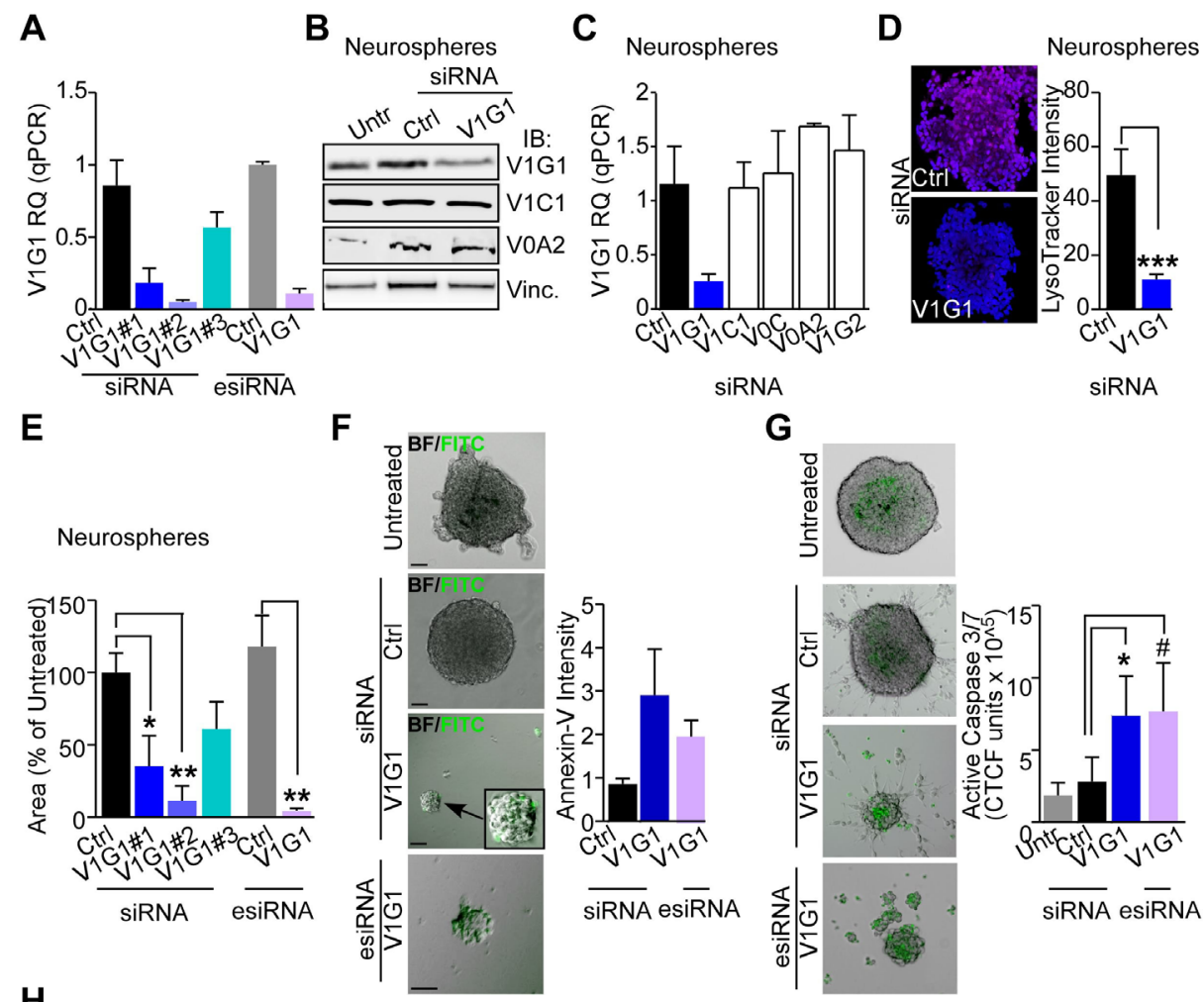

G
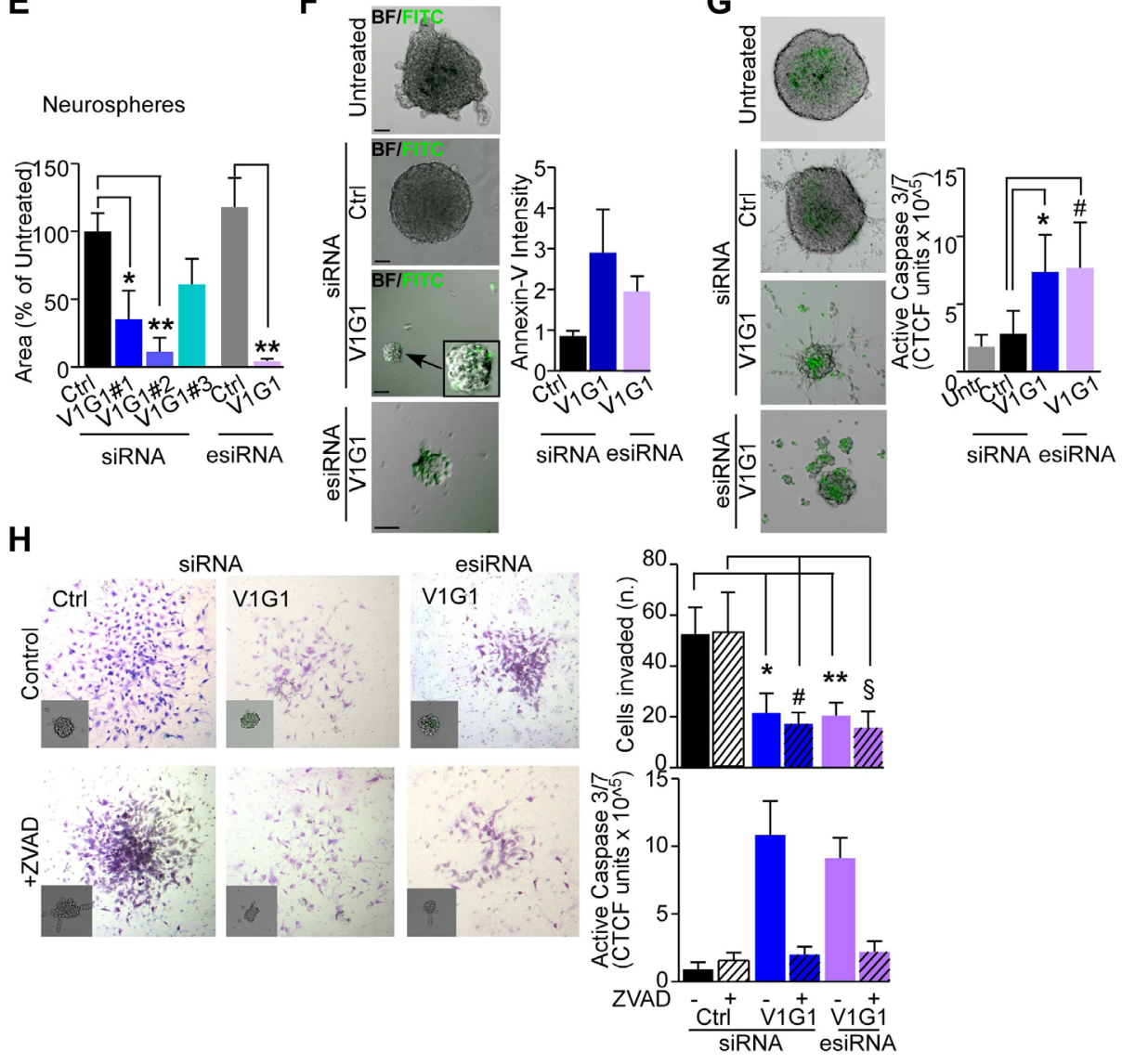

Figure 3: V-ATPase subunit G1 is central for GBM stem cell viability and invasive potential. A. Optimization of ATP6V1G1 transient knockdown in neurospheres by small interfering RNA (siRNA) was performed with three different targeting siRNAs or with esiRNA technology. The small interference RNA \#1 (V1G1\#1) was then chose for subsequent analyses together with V1G1-directed esiRNA unless otherwise indicated. B. Immunoblotting analysis of the indicated V-ATPase subunits in GBM neurospheres left untreated (Untr) or silenced with a control (Ctrl) or an ATP6V1G1-directed siRNA. A representative experiment is shown. C. ATP6V1G1 gene expression quantification in GBM neurospheres transfected with the indicated siRNA $(n=7)$. D. Confocal immunofluorescence images of live GBM neurospheres transfected with a non-targeting (Ctrl) or an ATP6V1G1-directed siRNA (V1G1) for 48 hours and then incubated with LysoTracker Red ${ }^{590}$ for 10 minutes at $37^{\circ} \mathrm{C}$. Maximum intensity projections are shown for a single representative experiment out of six repeats. Original magnification $\mathrm{x} 400$. Right, quantification of LysoTracker mean fluorescence intensity. ${ }^{* *}, p=0.01$ Mann-Whitney U test. E. GBM neurospheres were transfected with controls or targeting V1G1 siRNAs or esiRNA and their area was calculated 48 hours later. Neurospheres area is expressed relative to untreated cultures. F, G. Apoptosis induction or activation of caspase 3/7 after ATP6V1G1 knockdown (V1G1 siRNA or esiRNA) was evaluated by Annexin V staining or active caspase 3/7 probe and fluorescence microscopy, respectively. Right, Quantification of mean fluorescence intensities $(n=6)$. Scale bar indicates $100 \mu \mathrm{m}$. ${ }^{*}, p=0.016 ; \#, p=0.02$ by Mann-Whitney U test. H. Neurospheres silenced for ATP6V1G1 or with a scramble molecule (Ctrl) and pre-incubated with the general caspase inhibitor Z-VAD-FMK (ZVAD). or its negative control Z-FA-FMK (Control) were subjected to matrigel invasion for 24 hours after which invaded cells were stained with toluidine blue and counted. Representative images of an experiment out of six are shown. Insets, caspase activation was monitored by CellEvent Caspase 3/7 probe (green) by fluorescence microscopy and quantified as corrected total cell fluorescence (CTCF). Data are expressed as percentage of invaded spheres relative to the input. *, $p=0.02 ; \#, p=0.03 ; * *, p=0.004 ; \S$, $p=0.01$ by Mann-Whitney $\mathrm{U}$ test. 
even upon treatment with low dose of BafA1 (Figure 5E). Altogether, these data suggest that at high BafA1 doses cell death might occur through both apoptotic and necrotic mechanisms. Therefore, to avoid unspecific cell death, we used $20 \mathrm{nM}$ or $500 \mathrm{nM}$ of BafA1 for subsequent experiments. Confirming annexin $\mathrm{V}$ data and similar to what observed for ATP6V1G1 knockdown, BafA1 induced activation of Caspase 3/7 at both concentrations (Figure 5F). Besides inducing programmed cell death, BafA1 strongly inhibited motility of GBM neurospheres (Figure $5 \mathrm{G})$. Indeed, calcein AM/ToPro-3 differential staining of collagen-invading cells indicated that at $20 \mathrm{nM}$ migration is impaired in presence of very low level of dead cells, suggesting that V-ATPase inhibition might diminish GBM stem cells motility independently from cell death (Figure
$5 \mathrm{H})$.

Finally, impairment of clonogenic potential by BafA1 treatment inversely reflected V-ATPase G1 levels detected in patients' derived GMB neurospheres (Figure 5I; see Figure 4C for V1G1 levels). Conversely and strikingly, neither viability of differentiated GBM cell cultures (Supplementary Figure 10C-10E) or of commercial LN229 and T98G cells (Supplementary Figure 10F, 10G), nor motility of differentiated GBM cultures (Supplementary Figure $10 \mathrm{H}$ ) was affected by acute treatment with BafA1 at any dose. Overall these data indicate that V-ATPase pump function is required specifically by GBM neurospheres to preserve their tumorigenic potential and their propagating ability.
A

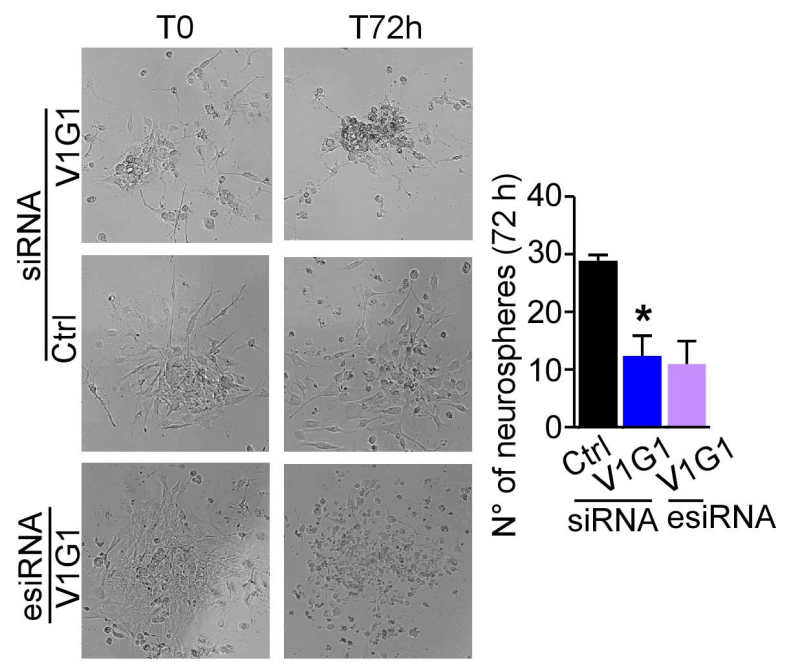

C

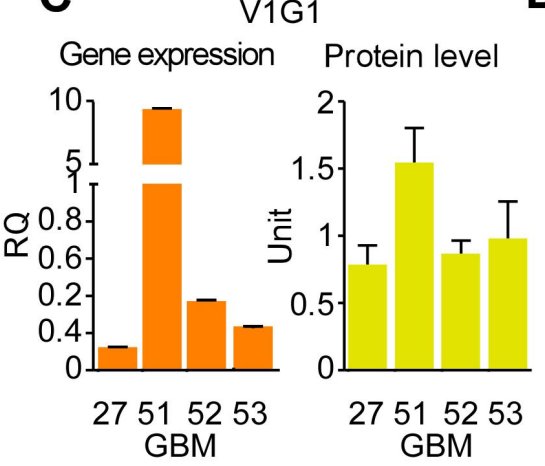

B

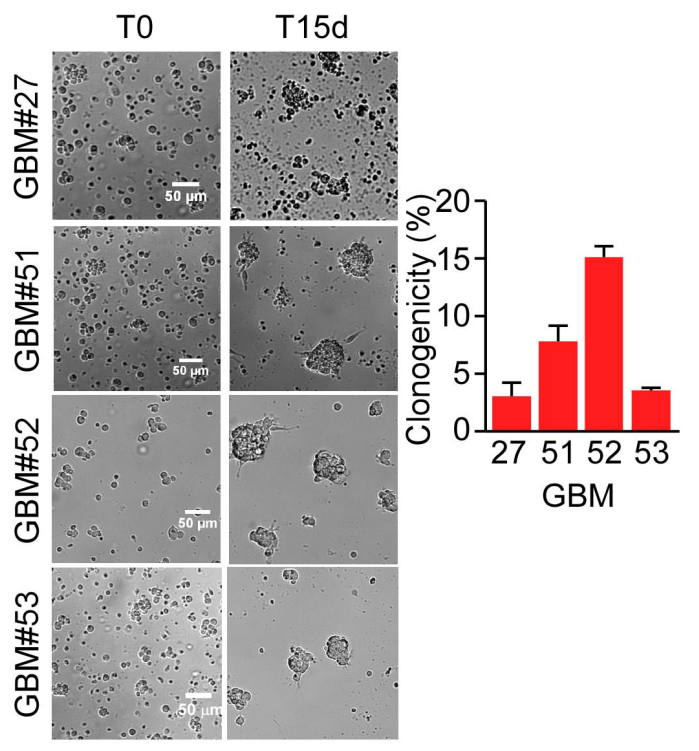

Figure 4: V-ATPase G1 levels are associated to clonogenicity of GBM neurospheres. A. The ability of GBM cultures to generate neurospheres after specific ATP6V1G1 knockdown (V1G1-siRNA or esiRNA) relative to control sample was evaluated by time lapse microscopy for 72 hours. Representative images captured from movies (Supplementary Movies 1-3) at time zero and at harvesting are shown. B. GBM neurospheres clonogenic capacity in methylcellulose containing media was analyzed in the indicated samples. Left, quantification of spheres containing at least 20 cells. C. V-ATPase G1 gene or protein expression levels in the indicated GBM cultures. D. The clonogenic potential of the indicated GBM neurosphere was assessed after V1G1 knockdown by siRNA or esiRNA and compared to corresponding controls. ${ }^{*}, p<0.05 ; * *, p<0.01$ by Mann Whitney $\mathrm{U}$ test. For all graphs, bars represent mean \pm SEM of at least six experiments. 


\section{V-ATPase inhibition decrease viability of GBM organotypic cultures}

To test the feasibility of V-ATPase inhibition in experimental set-up closest to the in vivo GBM situation, we used patient-derived organotypic glioma cultures which preserve the tri-dimensional tissue architecture and cellular heterogeneity of the original tumor [24, 25]. We therefore performed BafA1 treatment in a set of eight glioblastoma organotypic cultures (Supplementary Table 3), to compare drug effects on glioma viability and
A

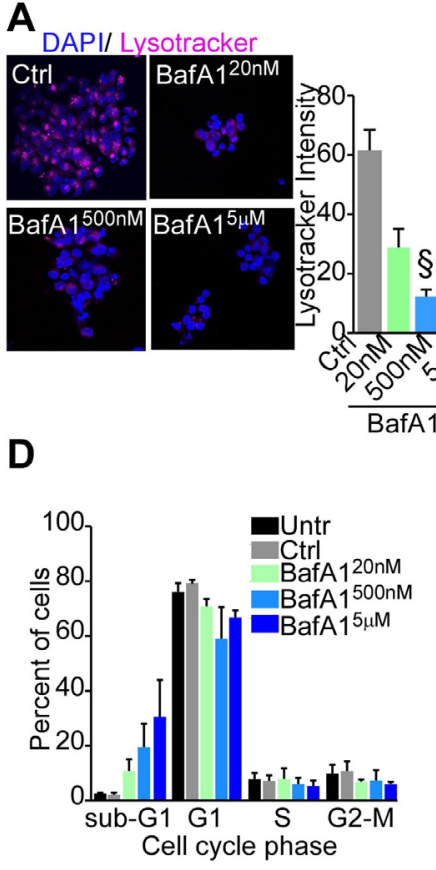

G

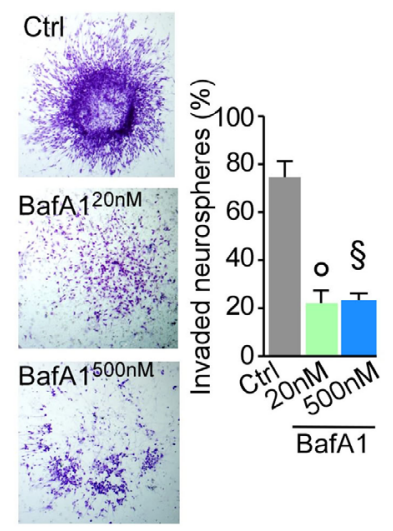

B

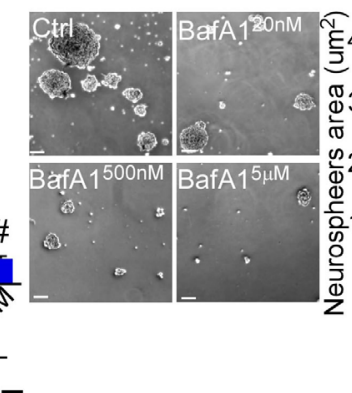

$E$

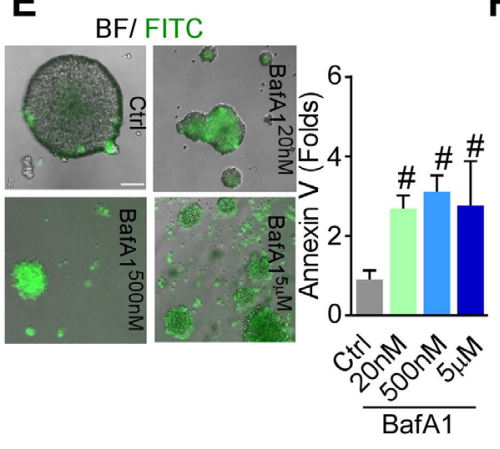

H
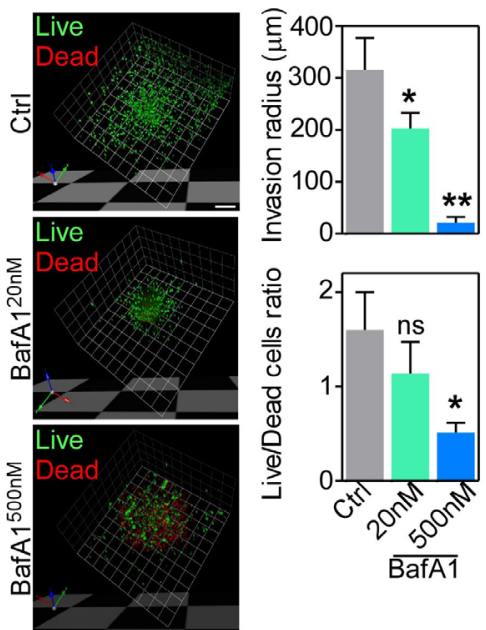

C
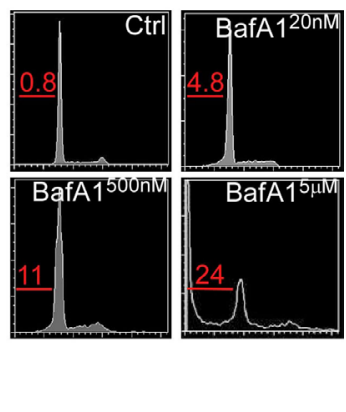

F
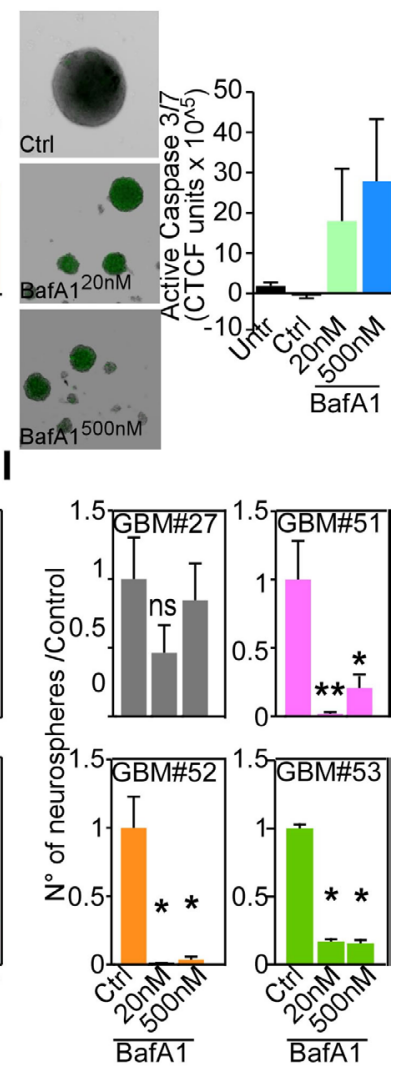

Figure 5: V-ATPase targeting through bafilomycin A1 abrogates GBM stem cells. A. Confocal immunofluorescence images of live GBM neurospheres treated with different concentration of bafilomycin A1 (BafA1) for 48 hours and then incubated with LysoTracker $\operatorname{Red}^{590}$ for 10 minutes at $37^{\circ} \mathrm{C}$. Maximum intensity projections are shown for a single representative experiment out of six repeats. Original magnification x 400. Right, quantification of mean fluorescence intensity. ${ }^{\S}, p=0.0004$; ${ }^{\#}, p=0.0001$. B.-F. GBM neurospheres treated with different doses of BafA1 for 48 hours were analyzed for dimensions B., DNA content by propidium iodide staining and flow cytometry C., D. induction of programmed cell death (Annexin V staining; E.), activation of caspase 3/7 by fluorescent microscopy $\mathbf{F}$., or cell invasion through matrigel by direct cell counting $\mathbf{G} . .{ }^{*}, p=0.016 ;{ }^{*}, p=0.03 ;{ }^{\circ}, p=0.013 ;{ }^{\S}, p=0.027$. For all graphs, bars represent mean \pm SEM of at least six experiments. H. GBM neurospheres were embedded in collagen and treated as indicated. After $48 \mathrm{~h}$ cultures were stained with calcein-AM (live cells, green) and ToPro-3 (dead cells, red) and analyzed by confocal microscopy. Right, Quantification of the invasion radius and of the live $v s$ dead cells ratio. Bars, $\operatorname{mean} \pm \operatorname{SEM}(n=4){ }^{*}, p=0.02 ; * *, p=0.004$. I. GBM neurospheres clonogenicity after BafA1 treatment was assessed by methylcellulose assay for the indicated cultures. Bars, mean $\pm \operatorname{SEM}(n=3)$. All statistics are from MannWhitney U test. 
proliferation with the alkylating agent temozolomide (TMZ), which is the standard of care for GBM. After 72 hours, treatment with BafA1 significantly decreased glioma proliferation (Ki67 labelling; $p=0.02$; Figure $6 \mathrm{~A}, 6 \mathrm{~B}$ ) and induced apoptosis (cleaved Caspase- $3^{\text {Asp } 175}$ expression; $p=0.03$; Figure $6 \mathrm{~A}, 6 \mathrm{C}$ ) while TMZ had little effect (Figure 6A-6C).

BafA1 and TMZ are known to interfere with autophagy, with TMZ inducing autophagosome formation and BafA1 blocking autophagosome fusion with lysosomes [26]. As previously documented in neuroblastoma cells in culture [27], treatment of GBM organotypic cultures with BafA1 increased lysosomeassociated membrane protein-1 (LAMP-1) content (Figure $6 \mathrm{D})$, indicating induction of lysosomal stress. However, levels of beclin1, a regulator of autophagosome formation, in GBM organotypic cultures treated with BafA1 did not change, suggesting that lysosomal stress did not involve
A

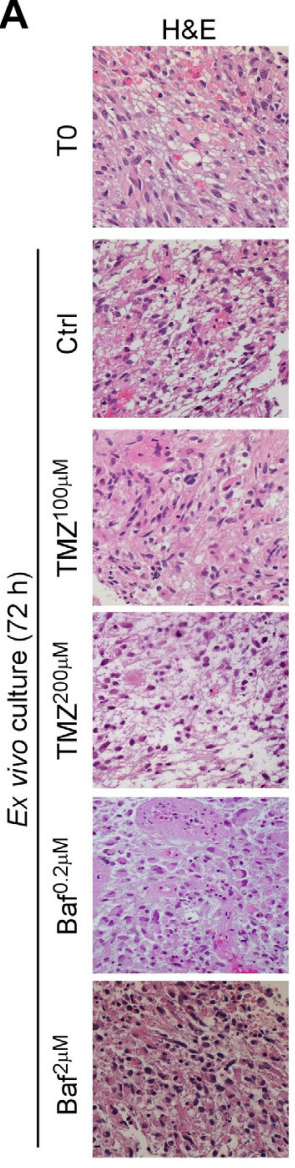

D

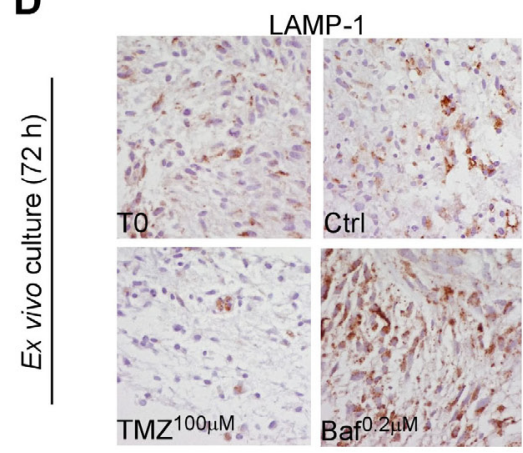

B

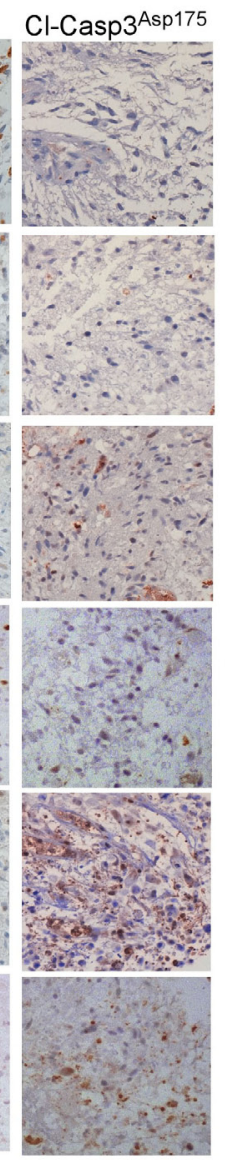

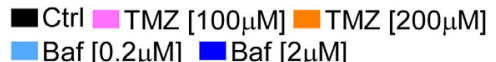

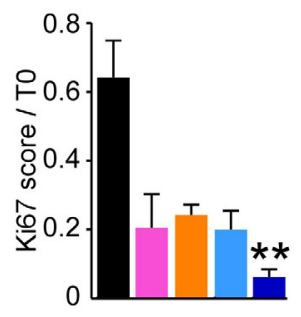

- Ctrl TMZ $[100 \mu \mathrm{M}]-\mathrm{TMZ}[200 \mu \mathrm{M}]$ Baf $[0.2 \mu \mathrm{M}] \square$ Baf $[2 \mu \mathrm{M}]$

C

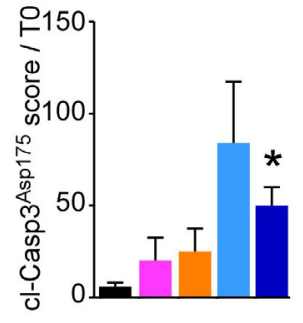

E

TMZ $[100 \mu \mathrm{M}] \quad \mathrm{TMZ}[200 \mu \mathrm{M}]$

Baf $[0.2 \mu \mathrm{M}] \quad$ Baf $[2 \mu \mathrm{M}]$

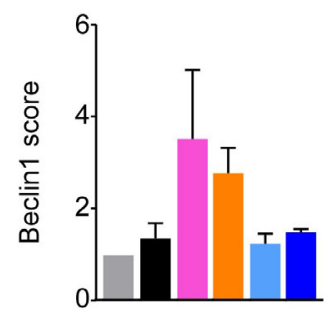

Figure 6: Bafilomycin A1 treatment decreases cell viability in GBM tissue cultures. Organotypic tissue cultures from eight GBMs were treated up to $72 \mathrm{~h}$ with different concentration of temozolomide (TMZ) or Bafilomycin A1 (Baf) and tissue proliferation (Ki67 labeling, A., B.), or apoptosis (Cleaved Caspase-3, A., C.) was scored in each sample relative to uncultured (T0) sample. *, $p=0.03$; **, $p$ $=0.007$ by Mann-Whitney U test. H\&E, Hematoxylin and Eosin. D., E. GBM tissue cultures treated as in A were analyzed for lysosomal content (LAMP-1, D.) or for expression of the autophagy-related protein Beclin 1 (E.) by immunohistochemistry. Photomicrographs show representative GBM cultures. Original magnification $\mathrm{x} 400$. Data are presented as mean $\pm \mathrm{SEM}$. 
modulation of tumor autophagy (Figure 6E). Therefore, our data do not support the possibility that autophagy is a mediator of cell death in GBM organotypic cultures treated with BafA1. Overall, these data indicate that $\mathrm{V}$-ATPase inhibition is more efficient than TMZ treatment to reduce viability of GBM tissue.

A

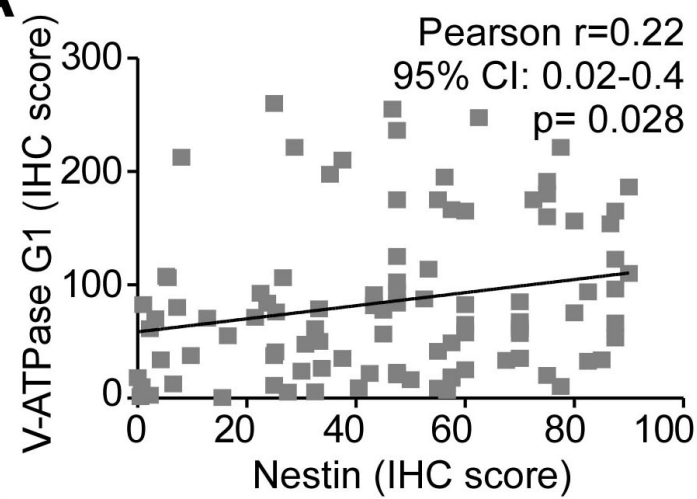

C

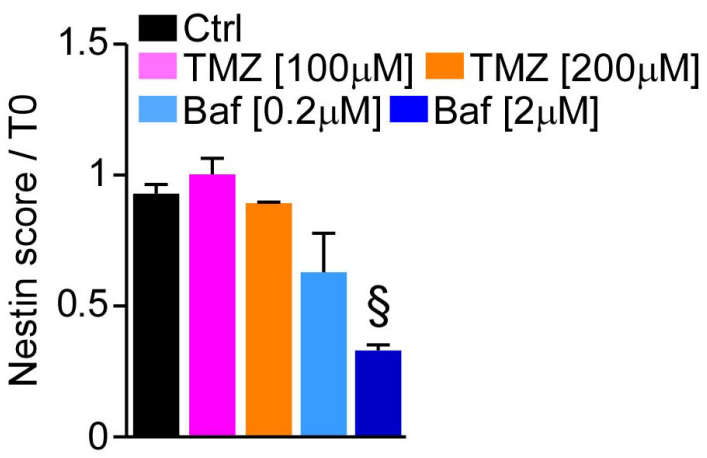

D

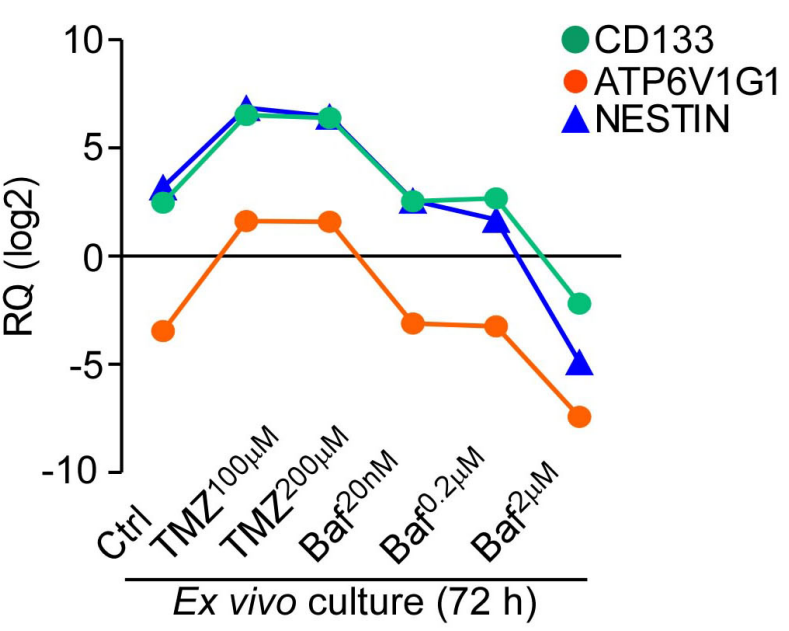

Treatment of GBM neurospheres or organotypic cultures with BafA1 induces selective stem cell depletion and reduces stem cell factors expression

Given the results obtained using BafA1 in GBM neurospheres and organotypic cultures, we finally assessed whether BafA1 treatment affected stem cells in GBM tissue. Using the glioma TMA platform (Table 1) we found

B

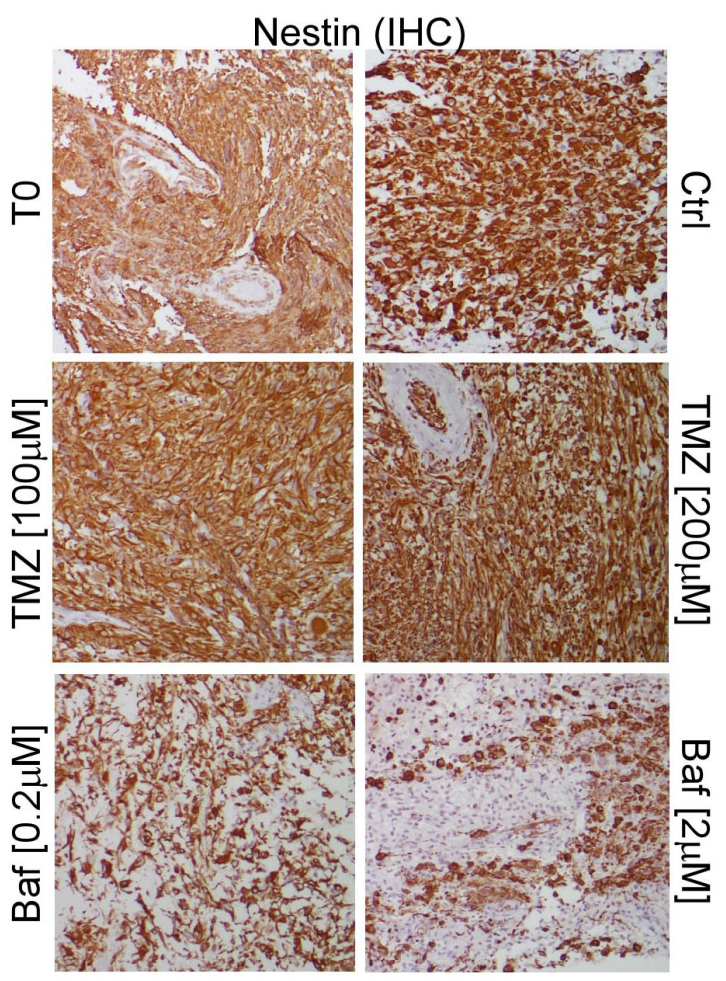

E

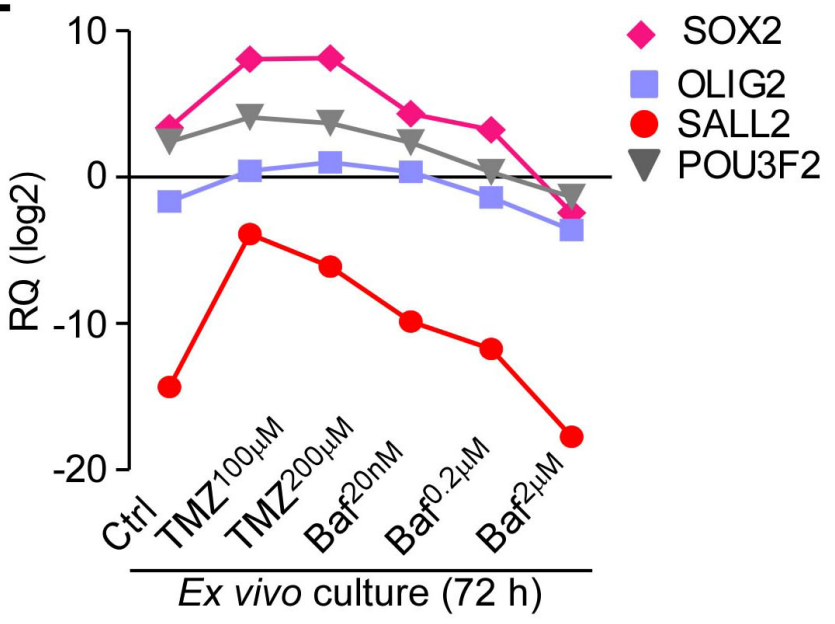

Figure 7: Bafilomycin A1 reduces stem cell factors expression in GBM organ cultures. A. Nestin immunoreactivity was analyzed in GBMs of the tissue micro-arrays and correlated to V-ATPase G1 expression in the same samples. B., C. Short term GBM organ cultures were treated as in Figure 6. Nestin presence was assessed after 72 hours and quantified relative to uncultured samples (T0). Original magnification $x 400$. Bars, mean \pm SEM. $\S, p=0.018$ by Mann-Whitney U test. D., E. The stem cell markers CD133, NESTIN or ATP6V1G1 (D.) and the neurodevelopmental transcription factors SOX2, OLIG2, SALL2 and POU3F2 (E.) were analyzed at gene expression level by qPCR in GBM organ cultures treated as indicated. Each point represents the mean of three cultures. RQ, mRNA Relative Quantity. 
that V-ATPase subunit G1 expression was significantly correlated with nestin in GBM $(n=98, p=0.028$; Figure 7A). In addition, treatment with BafA1, but not with TMZ, strongly reduced the number of nestin-positive cells in GBM organotypic cultures (Figure 7B, 7C). These data were confirmed at the gene expression level. Indeed, GBM organotypic cultures treated with the V-ATPase inhibitor displayed decreased levels of NESTIN and CD133 besides showing lesser expression of ATP6V1G1 (Figure 7D). We also analyzed a panel of transcription factors recently associated to GBM stem cell propagation, such as SOX2, OLIG2, SALL2 and POU3F2 [28]. While TMZ increased their expression in treated cultures, variable down-regulation of SOX2, SALL2 and POU3F2 was observed in GBM organotypic cultures incubated with $2 \mu \mathrm{M}$ of BafA1 (Figure 7E). Consistent with the effects observed in organotypic cultures, treatment of GBM neurospheres, which are known to be enriched in cancer stem cells [28], with BafA1 decreased nestin protein (Figure 8A) and mRNA levels (Figure 8B), as well as expression of stem cell-associated factors CD133, SOX2, SALL2 and POU3F2 to different degrees (Figure 8C, 8D), while V-ATPase G1 mRNA and protein levels remained substantially unchanged (Figure 8D). These data confirm that GBM stem cells are highly sensitive to V-ATPase inhibition.

\section{DISCUSSION}

In this study we show that V-ATPase G1 is a novel marker of poor prognosis for glioblastoma patients. Notably, V-ATPase G1 levels were predictive of overall survival in GBM patients independently of the epigenetic silencing of MGMT gene, IDH1 mutation, Karnofsky performance score, or patients' age at diagnosis. Our data

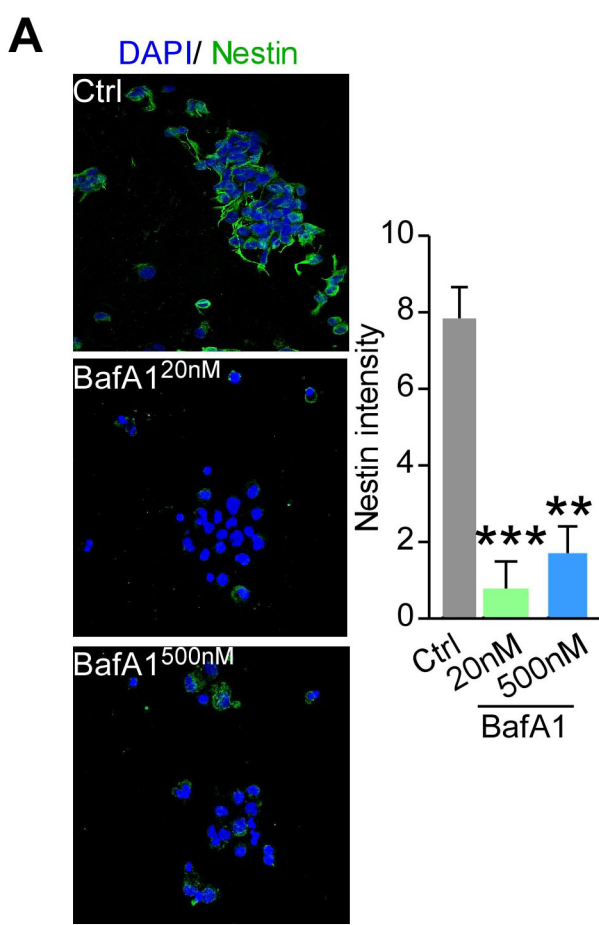

B

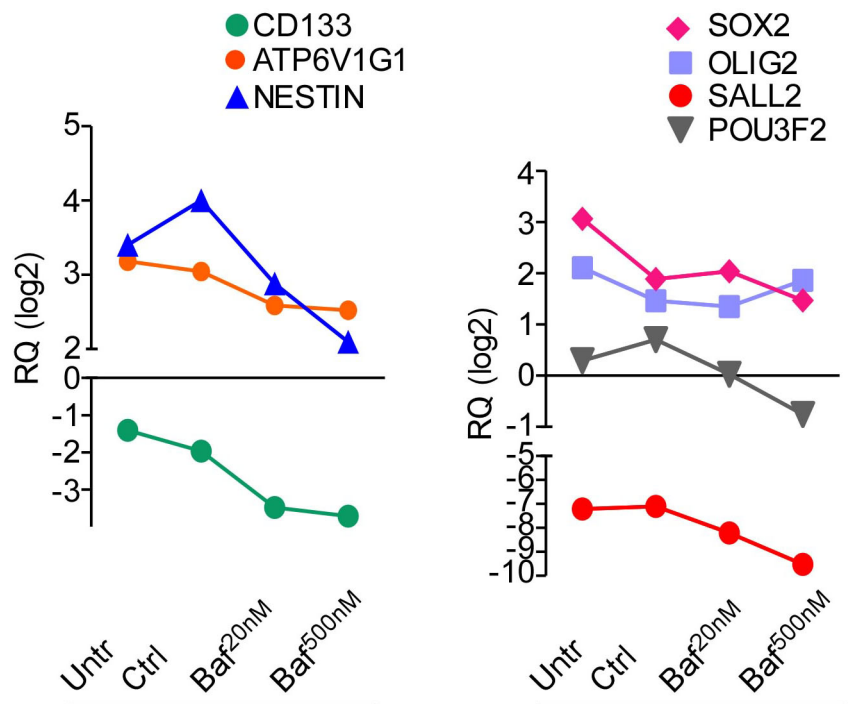

Neurospheres treatment $(48 \mathrm{~h})$ Neurospheres treatment $(48 \mathrm{~h})$

D

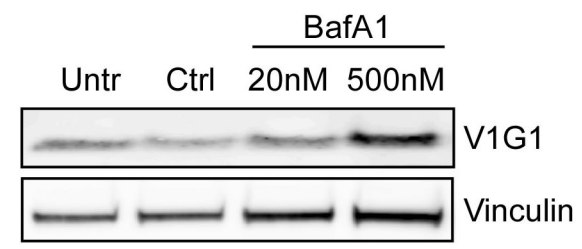

Figure 8: Bafilomycin A1 repress stemness features of GBM neurospheres. A. Nestin expression was evaluated by confocal immunofluorescence in GBM neurospheres incubated for 48 hours with vehicle or different concentrations of bafilomycin A1 and quantified in multiple z-stacks by ROI. ${ }^{* *}, p=0.007 ;{ }^{* * *}, p=0.0006$ by Mann-Whitney U test. Bars, mean $\pm \operatorname{SEM}(n=5)$. Left, Representative maximum intensity projection images of neurospheres stained for the indicated antibody. B., C. Gene expression evaluation of ATP6V1G1, CD133 and NESTIN, or of transcription factors involved in neurodevelopment and reprogramming (SOX2,OLIG2, SALL2 and POU3F2) in GBM neurospheres treated for 48 hours with $20 \mathrm{nM}$ or $500 \mathrm{nM}$ of bafilomycin A1, vehicle (Ctrl), or left untreated (Untr). Each point represents the mean of four experiments. RQ, mRNA Relative Quantity. D. V-ATPase G1 protein levels in GBM neurospheres after bafilomycin A1 treatment were analyzed by western blot. Vinculin was a loading control. 
reveal that ATP6V1G1 is selectively upregulated in GBM stem cells and that targeting of V-ATPase through specific gene knock-down or pharmacological inhibition hampers GBM viability, tumorigenicity and stem cell factors expression. We also demonstrate that V-ATPase inhibition by BafA1 is feasible and highly effective in human GBM organ cultures, an experimental platform that closely mimics the in vivo cancer situation [24, 25]. Given the selective exploitation of V-ATPase by glioma stem cells, our preliminary evidence suggests that V-ATPase targeting could represent an attractive novel therapeutic strategy in GBM, a fatal disease with no curative option. Indeed the suitability of targeting V-ATPase to eradicate cancer stem cells has been previously explored in embryonal rhabdomyosarcoma disease [22].

Overall, we found that V-ATPase G1 protein is progressively overexpressed by high-grade gliomas compared to less aggressive grade II tumors, whereas it is expressed at low level in normal brain. In our series, V-ATPase G1 was not associated to MGMT epigenetic status, but increased protein levels were detected in brain tumors wild-type for isocitrate dehydrogenase 1 (IDH1) enzyme, a known marker of worse prognosis [19], and this occurred irrespective of their grade. The oncogenic role of IDH1 mutations in gliomas is unclear. Nevertheless, IDH enzymes are involved in tumor metabolism and energy (NADP + ) production [29]. Our findings may suggest that a regulatory loop between an acidic microenvironment and altered bioenergetics in tumor cells could contribute to tumorigenesis. Further work will be needed to understand the mechanism behind the correlation between V-ATPase and IDH enzymes in glioma cells.

Little is known about regulation of V-ATPase subunits expression during tumorigenesis. A previous report indicates that V-ATPase G1 is expressed ubiquitously in mouse brain, while its paralog V-ATPase subunit G2 is expressed in neurons of the central nervous system where it is specifically involved in acidification of synaptic vesicles [30]. V-ATPase upregulation has been described to induce cell transformation [10], to sustain the cancer stem cell niche [22], and as a defensive mechanism adopted by cancer cells to escape apoptosis induced by anticancer agents [31]. Specifically, V-ATPase activity appears to be required by ovarian or breast cancer cells to invade and metastasize $[15,23,32-34]$, or to activate oncogenic signaling [5, 35]. Finally, ATP6V0A encoding a specific subunit associated to V-ATPase function at the plasma membrane, was found upregulated in grade III gliomas [12]. Despite this, the functional relevance of the upregulation of V-ATPase subunits in human cancer is unclear. However, we showed that pharmacologic inhibition of V-ATPase function impact tumor viability and GBM stem cell features, suggesting a clear requirement for pump activity. In good agreement, a recent investigation reported that targeting V-ATPase pump activity hampers growth and tumorigenic potential of rhabdomyosarcoma CSC [22]. In such system, high V-ATPase activity was associated to increased invasive ability and reduced sensitivity to chemotherapeutics, as previously reported [8, 14, 23]. Interestingly, similar to V-ATPase the pH-dependent CLIC-1 channel [36] is required for proliferation of GBM stem cells [18, 21], suggesting that $\mathrm{pH}$ unbalances might contribute to GBM stem cell niche maintenance. Altogether, this evidence indicates that V-ATPase might control multiple aspects of tumor development by mechanisms that await elucidation.

In human brain V-ATPase G1 immunostaining strongly resembled nestin pattern in either human glioma or normal tissues. In normal brain or grade II gliomas, both V-ATPase G1 and nestin localize to endothelial cells of blood vessels [37, 38]. Conversely, high-grade gliomas displayed V-ATPase G1 and nestin upregulation in tumor cells, and expression of both proteins was decreased by BafA1. These preliminary data suggest that V-ATPase might be involved in acquisition and/or maintenance of the GBM cancer stem cell niche. Consistent with this, BafA1 has been shown to sensitize glioma stem cells to gamma-radiation reducing their viability and ability to form neurospheres [39].

Concluding, our results suggest that high levels of ATP6V1G1 are a characteristic of GBM stem cells and could influence the onset of chemoresistance typical of high grades gliomas. Indeed patients with a low V-ATPase subunit G1 expression had a longer OS and a better response to adjuvant concomitant chemo-radiation therapy in terms of time to disease progression. Despite increased understanding of the molecular alterations that characterize different subsets of high-grade glioma, almost all patients face a fatal disease with inadequate treatment options. Therefore, there is a crucial need to identify proteins that could be effectively targeted [18]. In this scenario, we have shed light on a core aspect of the cellular machinery that is selectively exploited by GBM cancer stem cells, whose activity can be modulated pharmacologically. Considering that V-ATPase directed drugs are currently under preclinical development [14, 15], our work constitutes the groundwork for future understanding of the antitumor activity of V-ATPase inhibition in gliomas.

\section{MATERIALS AND METHODS}

\section{Patients}

For this investigation, two series of patients diagnosed with de novo glioma were enrolled at Fondazione IRCCS Ca' Granda Ospedale Maggiore Policlinico (Milan, Italy). A first series consisted of 54 patients that underwent surgery between 2013 and 2014, of which both fresh and archival neoplastic tissue 
could be retrieved for molecular or histological analyses respectively (Gene expression series). A second set of 187 patients that consecutively underwent surgery between 2008 and 2010 was enrolled for retrospective investigations on formalin-fixed paraffin-embedded tissues (Tissue microarray series) as previously described [40]. All patients were treated with surgical resection of the lesion and nobody received a merely diagnostic procedure. The Institutional Review Board approved the study. Gliomas were staged according to the WHO classification [41] and clinicopathological or molecular characteristics of both patients' series are described in Table 1. Following histological diagnosis, all patients affected by high grade gliomas (HGG; grades III and GBM) underwent concomitant chemo-radiation therapy according to the Stupp's protocol [42]. Follow-up data was available for GBM patients and it consisted of neurooncological assessment and brain MRI with gadolinium every 3 months as described [40]. Tumor progression was defined according to MacDonald's criteria [17]. Time to progression (TTP) and overall survival (OS) records were available for $69(70 \%)$ and 70 (71\%) GBM patients, respectively. TTP was calculated from surgery to tumor progression determined by radiological (brain MRIs) procedures, whereas OS was calculated from surgery to patients' death. Follow-up ended in December 2012.

Tissue microarrays (TMAs) of glioma or normal brain tissues were created as previously described [43], with slight modifications. Detailed procedures for TMAs construction and immunohistochemical (IHC) experiments are detailed in the Supplementary Information.

\section{Oncomine analysis}

Differential V-ATPase subunits expression was analyzed in published microarray data sets using a Webbased data-mining platform (Oncomine; available at http://www.oncomine.org/). Relative expression data of V-ATPase subunits were collected from brain cancer datasets only. The $t$-statistic for significant comparison is provided within the data sets in Oncomine.

\section{Glioblastoma primary cultures and neurospheres}

GBM neurospheres or differentiated monolayer cultures (Supplementary Figure 4B-4D) were obtained as described [18, 44] from seven chemo- and radionaïve patients surgically treated at Neurosurgery Division (Fondazione IRCCS Ca' Granda Ospedale Maggiore Policlinico) and histologically diagnosed with glioblastoma (WHO grade IV, GBM). Differentiated primary cultures were also derived from two patients diagnosed with grade I glioma (Supplementary Table 1). Post-surgical samples were used after patients' informed consent and Institutional Ethical Committee approval and primary cultures were coded as Grade I\#1, 2 and GBM with case number (Supplementary Table 1). Details of this procedure can be found online in the Supplementary Information.

\section{Ex-vivo glioma tissue cultures}

Glioma tissue slices were obtained from surgical waste of eight patients affected by GBM treated at Fondazione IRCCS Ca' Granda Ospedale Maggiore Policlinco (Milan, Italy). Written informed consent was obtained from all patients. Clinicopathological characteristics are described in Supplementary Table 3. Briefly, precise thick $(300 \mu \mathrm{m})$ tissue sections were generated using a vibratome (VT1200, Leica Microsystems, Milan, Italy) as previously described [24, 25 ], and cultivated up to 72 hours in presence or absence of $100 \mu \mathrm{M}$ or $200 \mu \mathrm{M}$ temozolomide, $0.2 \mu \mathrm{M}$ or $2 \mu \mathrm{M}$ bafilomycin A1 (both drugs were from Sigma-Aldrich, Milan, Italy). As control sample we used an untreated tissue slice cultivated in complete media supplemented with vehicle ( $1 \mu \mathrm{l}$ of DMSO in $1 \mathrm{ml}$ of media). Culturing medium supplemented with drugs or vehicle was replaced every $24 \mathrm{~h}$. At harvesting, tissue cultures were snap-frozen or formalin-fixed and paraffin-embedded (FFPE) for molecular or morphological (hematoxylin and eosin staining) and immunohistochemistry analyses, respectively. For each glioma culture, tissue proliferation (Ki67 labeling), apoptosis (cleaved Caspase-3 Asp175), Beclin 1, LAMP-1, Nestin or V-ATPase G1 (clone D-5; Santa Cruz Biotech, Santa Cruz, CA, USA) protein presence and expression in cultivated tissue slices were evaluated by immunohistochemistry (IHC) and scored as percentage of positive cells out of the total. IHC scores in cultivated tissue slices are expressed relative to baseline uncultured sample (T0). Similarly, selected genes expression (CD133, NESTIN, ATP6V1G1, POU3F2, SALL2, OLIG2, SOX2; Supplementary Table 4) were quantified relative to reference gene (18S rRNA) in cultured or not samples. Detailed immunohistochemistry and gene expression protocols can be found in the Supplementary Information.

\section{RNA purification and qPCR}

Total RNA was purified from glioma tissues or cell cultures using Trizol reagent (Life Technologies Inc.) or Master Pure RNA purification kit (Epicentre Biotechnologies, Illumina; Madison, WI, USA) following manufacturer procedures. Detail of retrotranscription or quantitative PCR reactions are provided in the Supplementary Information. 


\section{Cell lines, siRNA transfections and V-ATPase inhibition}

SW1088, T98G or LN229 glioma cell lines were purchased from ATCC (Manassas, VA, USA) and cultured as suggested by the manufacturer. Protein extract from E18 primary rat hippocampal neurons (E18 RHN) were a generous gift from Fabio Grohovaz lab (IRCCS San Raffaele Scientific Institute, Neuroscience Division, Milan, Italy). GBM primary cultures or commercial cell lines were transiently transfected in Optimem media using Lipofectamine 3000 (both from Invitrogen, Life Technologies Inc.) and 100pM of specific or nontargeting control siRNAs or esiRNA (all from Sigma Aldrich, St. Louis, MO, USA) as described [45]. Three ATP6V1G1 siRNAs were initially tested (MISSION siRNA SASI_Hs02_00338522; SASI_Hs01_00227261; SASI_Hs01_00227268) together with ATP6V1G1targeting esiRNA (MISSION esiRNA EHU156241) and corresponding non-targeting control (MISSION esiRNA EHUEGFP). The siRNA SASI_Hs02_00338522 (siRNA-V1G1\#1) and V1G1-esiRNA were then chosen for functional experiments if not otherwise indicated. After five hours, transfection mixture was replaced with standard culturing media. V-ATPase inhibition was achieved treating cultures with different concentrations of bafilomycin A1 (BafA1, range 10nM-5 $\mu \mathrm{M}$; Sigma Aldrich) or concanamycin A (ConcA, range $50-500 \mathrm{nM}$; Sigma Aldrich) for 48 hours. Effect of siRNAs or drugs incubation on V-ATPase pump activity was measured incubating primary or commercial GBM cultures with $1 \mu \mathrm{M}$ of LysoTracker Red (DND-99, Life Technologies Inc.) for $10 \mathrm{~min}$ or with $1 \mu \mathrm{M}$ of DQ-Red BSA (Life Technologies Inc.) for $1 \mathrm{~h}$, as described [35]. Then cultures were rinsed and mounted immediately (Lysotracker) or fixed with 4\% paraformaldehyde (4\% PFA; DQ-BSA) for confocal microscopy examination (TCS SP5 Confocal, Leica Microsystems, Milan, Italy).

\section{Cell viability assays}

LN229 or T98G cell viability after V-ATPase inhibition by selective drugs was analyzed after $48 \mathrm{~h}$ by measuring mitochondrial function through the reduction of 3-(4,5-dimethylthiazol-2-yl)-2,5-diphenyltetrazolium bromide (MTT, Sigma-Aldrich) as reported [45]. Cell cycle transitions and quantification of hypodiploid DNA content (i.e. sub-G1 fraction) were determined in transfected or drug treated cultures after $48 \mathrm{~h}$, by propidium iodide staining and flow cytometry, as described [45]. Alternatively, cells were stained with Annexin V/PI using the FITC Annexin V Apoptosis Detection Kit (BD Bioscience, San Diego, CA, USA), evaluated by multiparametric flow cytometry and analyzed using FlowJo software. For GBM neurospheres the FITC
Annexin V Fluorescence Microscopy Kit (BD Bioscience) was used and signal intensity was acquired using a fluorescence inverted microscopy (DMI4000B, Leica Microsystems). Detection of effectors caspase activation was performed incubating live cultures with the CellEvent Caspase-3/7 Green probe (Life Technologies) and signal (at $503 \mathrm{~nm}$ ) or bright-field images were acquired using a fluorescence microscope. Details of the procedure can be found in the Supplementary Information.

\section{Cell motility and cell invasion}

LN229 cells grown in adhesion were transfected with ATP6V1G1 siRNA or control molecule for $24 \mathrm{~h}$ after which $5 \times 104$ cells were seeded in serum-free medium on collagen- or Matrigel-coated chambers (BD Biosciences) to evaluate cell migration or invasion, respectively. For GBM neurospheres invasion assay, an average of 13 spheres per condition (untreated, vehicle, bafilomycin A1treated samples or control- and ATP6V1G1 knockdown samples) was used. For ATP6V1G1 or control-silenced glioma cultures the standard culturing medium was used as chemoattractant in the bottom chamber. When bafilomycin A1 effect was tested, complete Neurocult media (Stemcell Technologies) supplemented with the indicated concentration of the drug was used as chemoattractant. Cells or neurospheres were allowed to migrate or invade for $24 \mathrm{~h}$, as described [45] after which cultures present on lower insert surface were fixed with methanol, stained with toluidine blue, and quantified by direct cell counting using bright-field optical microscopy (Leica DMI4000B). For neuropsheres, the number of invaded foci was normalized on the original input. GBM neurospheres invasion experiment was repeated six times in duplicate using six different GBM cultures, whereas other motility experiments were performed three times in duplicate. When neurospheres were preincubated with the general caspase inhibitor Z-VAD-FMK (ZVAD), or its negative control inhibitor Z-FA-FMK (Control; both from BD Pharmingen), $10 \mu \mathrm{M}$ of the indicated molecule was added to the cultures one hour before the seeding on matrigel inserts. Effective inhibition of active caspase 3/7 was analyzed using CellEvent caspase 3/7 fluorescent probe (Life Technologies) in parallel plates when matrigel inserts were harvested. For analysis of contextual tumor cell viability and motility, neurospheres were embedded in $600 \mu \mathrm{l}$ of bovine collagen type I (Sigma Aldrich) in 24-well plates, overlaid with $1 \mathrm{ml}$ of growth medium containing $20 \mathrm{nM}$ of bafilomycin A1 or vehicle (DMSO, control). After $48 \mathrm{~h}$, neurospheres were analyzed for changes in maximum invasion distance (radius) and quantification of live versus dead cells was performed staining the spheroids with calcein-AM (live, green) and ToPro-3 (dead, red; both reagents were from Life Technologies) as described [46]. Samples were imaged with a HCX PL Fluotar 10X/0.0NA objective using 
a Leica TCS SP5 confocal microscope, and multiple stacks (Z-step $=4 \mu \mathrm{m})$ were generated in 2 channels. 3-D reconstruction of stained neurospeheres was performed using Volocity 3D Image analysis software (PerkinElmer, Waltham, MA, USA).

\section{Immunofluorescence and time-lapse microscopy}

For immunofluorescence experiments, glioma monolayer cultures were grown on cover-glasses whereas neurospheres were cyto-spinned on charged slides (Thermo Scientific, Waltham, MA, USA). Glioma cultures were stained with primary antibodies against V-ATPase G1 (D5, Santa Cruz Biotechnologies), GFAP (G9269; Sigma Aldrich), cleaved Caspase-3 (D175; Cell Signaling Technologies, Danvers, MA, USA), Nestin (MAB 1259; R\&D Systems, Abingdon, UK), Sox2 (D6D9, Cell Signaling Technology Inc.), Oligodendrocyte marker O4 (O7139; Sigma Aldrich), GFAP (G9269; Sigma Aldrich), or Tuji1 (T3952; Sigma Aldrich). Detailed procedures are available in the Supplementary Information. Confocal images were generated with a Leica TCS SP5 Confocal microscope (Leica Microsystems). For each sample, analyses of fluorescence intensities was performed in at least five randomly picked fields per condition using ImageJ software (imagej.nih.gov/ij) and experiments were repeated three or 6 times for monolayers cultures or GBM neurospheres, respectively. For Annexin V or Lysotracker quantification the fluorescence threshold was set, and the mean intensity of the fluorochrome was calculated and plotted in the graph. For proteins staining quantification, the area of the cells or the spheres was defined using a ROI as a boundary. We calculated the integrated intensity of the whole ROI. Detailed procedures can be found in the Supplementary Information section. Time-lapse microscopy experiments were performed using three primary GBM cell monolayers cultivated in stem cell permissive media in 48-wells plates. Briefly, GBM cultures at the second or third culturing passage were transfected with a control- or ATP6V1G1-directed siRNA (Sigma Aldrich) as described above, or left untreated. Then GBM cells were monitored over a period of 72 hours for spheres formation using a time-lapse microscope (Eclipse Ti-E, Nikon Instruments, Florence, Italy). Images for each sample were snapped every two hours and then assembled to a movie in QuickTime format.

\section{Statistical analysis}

Groups' comparisons were performed using unpaired two-sided Student's $t$ test (with Welch's correction if groups had unequal variances) or MannWhitney $U$ test when appropriate (GraphPad Prism software, La Jolla, CA, USA). The significance of a variable for GBM patients' prognosis was analyzed using the Cox regression hazard model either as univariate or multivariate analysis (MedCalc software, Mariakerke, Belgium) considering proteins IHC scores, MGMT methylation status, age at diagnosis, presence of IDH1 ${ }^{\mathrm{R} 132 \mathrm{H}}$ mutation or tumor grade as categorical variables. To generate a cut-off for V-ATPase G1 IHC score we used receiver operating characteristics (ROC) curves and the non-arbitrary criterion derived from the Youden index ( $\mathrm{J}$, MedCalc Software) as described [40]. Glioma patients were then sorted into low or high-expressor categories. The Kaplan Meier method was used to plot survival curves of glioblastoma patients. In TTP analysis patients' death were censored. Difference in survival curves was computed using the Log Rank test (MedCalc Software).

Two-sided $P$ values less than 0.05 were considered statistically significant.

\section{ACKNOWLEDGMENTS}

This work was supported by Fondazione Cariplo (2014-1148 to VV), Fondazione IRCCS Ca' Granda and Fondazione INGM - Grant in Molecular Medicine 2014 (to VV), and from Ministero della Salute "Ricerca Corrente Program" (to PR). T.V. is supported by AIRC and Telethon Foundations. MR, GG and IB are supported by a fellowship from the Doctorate School of Molecular and Translational Medicine at University of Milan. The authors are thankful to Barbara Bettegazzi for providing reagents (Fabio Grohovaz lab, Neuroscience Division, IRCCS San Raffaele Scientific Institute, Milan, Italy), to Massimilano Garrè and to Dario Parazzoli (Imaging Unit, IFOM-IEO Campus) for help with 3D models of tumorcell invasion.

\section{CONFLICTS OF INTEREST}

All Authors declare that no conflict of interests exists.

\section{REFERENCES}

1. Nishi T, Forgac M. The vacuolar $(\mathrm{H}+)$-ATPases.-Nature's most versatile proton pumps. Nature Reviews Molecular Cell Biology. 2002; 3: 94-103.

2. Marshansky V, Futai M. The V-type H+-ATPase in vesicular trafficking: targeting, regulation and function. Current Opinion in Cell Biology. 2008; 20: 415-426.

3. Cruciat C-M, Ohkawara B, Acebron SP, Karaulanov E, Reinhard C, Ingelfinger D, Boutros M, Niehrs C. Requirement of Prorenin Receptor and Vacuolar H+ATPase-Mediated Acidification for Wnt Signaling. Science. 2010; 327: 459-463.

4. Lange C, Prenninger S, Knuckles P, Taylor V, Levin M, Calegari F. The H+ Vacuolar ATPase Maintains Neural Stem Cells in the Developing Mouse Cortex. Stem Cells 
and Development. 2011; 20: 843-850.

5. Vaccari T, Duchi S, Cortese K, Tacchetti C, Bilder D. The vacuolar ATPase is required for physiological as well as pathological activation of the Notch receptor. Development. 2010; 137: 1825-1832.

6. Yan Y, Denef N, Schüpbach T. The Vacuolar Proton Pump, V-ATPase, Is Required for Notch Signaling and Endosomal Trafficking in Drosophila. Developmental Cell. 2009; 17: 387-402.

7. Zoncu R, Bar-Peled L, Efeyan A, Wang S, Sancak Y, Sabatini DM. mTORC1 Senses Lysosomal Amino Acids Through an Inside-Out Mechanism That Requires the Vacuolar H+-ATPase. Science. 2011; 334: 678-683.

8. Chiche J, Brahimi-Horn MC, Pouysségur J. Tumour hypoxia induces a metabolic shift causing acidosis: a common feature in cancer. Journal of Cellular and Molecular Medicine. 2010; 14: 771-794.

9. Stock C, Schwab A. Protons make tumor cells move like clockwork. Pflügers Archiv - European Journal of Physiology. 2009; 458: 981-992.

10. Petzoldt AG, Gleixner EM, Fumagalli A, Vaccari T, Simons M. Elevated expression of the V-ATPase C subunit triggers JNK-dependent cell invasion and overgrowth in a Drosophila epithelium. Disease Models \& Mechanisms. 2013; 6: 689-700.

11. Webb BA, Chimenti M, Jacobson MP, Barber DL. Dysregulated $\mathrm{pH}$ : a perfect storm for cancer progression. Nature Reviews Cancer. 2011; 11: 671-677.

12. Gleize V, Boisselier B, Marie Y, Poëa-Guyon S, Sanson $\mathrm{M}$, Morel N. The renal v-ATPase a4 subunit is expressed in specific subtypes of human gliomas. Glia. 2012; 60: 10041012.

13. Kapoor S. Bafilomycin A1 and its attenuating effect on tumour growth in systemic malignancies, especially gastrointestinal malignancies. Clinical Science. 2013; 125: 219.

14. Pérez-Sayáns M, Somoza-Martín JM, Barros-Angueira F, Rey JMG, García-García A. V-ATPase inhibitors and implication in cancer treatment. Cancer Treatment Reviews. 2009; 35: 707-713.

15. Wiedmann RM, Von Schwarzenberg K, Palamidessi A, Schreiner L, Kubisch R, Liebl J, Schempp C, Trauner D, Vereb G, Zahler S, Wagner E, Müller R, Scita G, et al. The V-ATPase-Inhibitor Archazolid Abrogates Tumor Metastasis via Inhibition of Endocytic Activation of the Rho-GTPase Rac1. Cancer Research. 2012; 72: 5976-5987.

16. von Schwarzenberg K, Wiedmann RM, Oak P, Schulz S, Zischka H, Wanner G, Efferth T, Trauner D, Vollmar AM. Mode of cell death induction by pharmacological vacuolar H+-ATPase (V-ATPase) inhibition. J Biol Chem. 2013; 288: 1385-1396.

17. Grimm SA, Chamberlain MC. State of the art and perspectives in the treatment of glioblastoma. CNS Oncology. 2012; 1: 49-70.
18. Setti M, Savalli N, Osti D, Richichi C, Angelini M, Brescia P, Fornasari L, Carro MS, Mazzanti M, Pelicci G. Functional role of CLIC1 ion channel in glioblastomaderived stem/progenitor cells. J Natl Cancer Inst. 2013; 105: 1644-1655.

19. Boots-Sprenger SHE, Sijben A, Rijntjes J, Tops BB, Idema AJ, Rivera AL, Bleeker FE, Gijtenbeek AM, Diefes K, Heathcock L, Aldape KD, Jeuken JW, Wesseling P. Significance of complete $1 \mathrm{p} / 19 \mathrm{q}$ co-deletion, IDH1 mutation and MGMT promoter methylation in gliomas: use with caution. Modern Pathology. 2013; 26: 922-929.

20. Dunn GP, Rinne ML, Wykosky J, Genovese G, Quayle SN, Dunn IF, Agarwalla PK, Chheda MG, Campos B, Wang A, Brennan C, Ligon KL, Furnari F, et al. Emerging insights into the molecular and cellular basis of glioblastoma. Genes \& Development. 2012; 26: 756-784.

21. Gritti M, Würth R, Angelini M, Barbieri F, Peretti M, Pizzi E, Pattarozzi A, Carra E, Sirito R, Daga A, Curmi PM, Mazzanti M, Florio T. Metformin repositioning as antitumoral agent: selective antiproliferative effects in human glioblastoma stem cells, via inhibition of CLIC1mediated ion current. Oncotarget. 2014; 5: 11252-11268.

22. Salerno M, Avnet S, Bonuccelli G, Hosogi S, Granchi D, Baldini N. Impairment of lysosomal activity as a therapeutic modality targeting cancer stem cells of embryonal rhabdomyosarcoma cell line RD. PLoS One. 2014; 9: e110340.

23. Kulshrestha A, Katara GK, Ibrahim S, Pamarthy S, Jaiswal MK, Sachs AG, Beaman KD. Vacuolar ATPase 'a2' isoform exhibits distinct cell surface accumulation and modulates matrix metalloproteinase activity in ovarian cancer. Oncotarget. 2015; 6: 3797-810.

24. Vaira V, Fedele G, Pyne S, Fasoli E, Zadra G, Bailey D, Snyder E, Faversani A, Coggi G, Flavin R, Bosari S, Loda M. Preclinical model of organotypic culture for pharmacodynamic profiling of human tumors. Proc Natl Acad Sci U S A. 2010; 107: 8352-8356.

25. Faversani A, Vaira V, Moro GP, Tosi D, Lopergolo A, Schultz DC, Rivadeneira D, Altieri DC, Bosari S. Survivin family proteins as novel molecular determinants of doxorubicin resistance in organotypic human breast tumors. Breast Cancer Res. 2014; 16: R55.

26. Kanzawa T, Germano IM, Komata T, Ito H, Kondo Y, Kondo S. Role of autophagy in temozolomide-induced cytotoxicity for malignant glioma cells. Cell Death Differ. 2004; 11: 448-457.

27. Mangieri LR, Mader BJ, Thomas CE, Taylor CA, Luker AM, Tse TE, Huisingh C, Shacka JJ. ATP6V0C knockdown in neuroblastoma cells alters autophagy-lysosome pathway function and metabolism of proteins that accumulate in neurodegenerative disease. PLoS One. 2014; 9: e93257.

28. Suvà $\mathrm{ML}$, Rheinbay $\mathrm{E}$, Gillespie $\mathrm{SM}$, Patel $\mathrm{AP}$, Wakimoto H, Rabkin SD, Riggi N, Chi AS, Cahill DP, Nahed BV, Curry WT, Martuza RL, Rivera MN, et al. Reconstructing and reprogramming the tumor-propagating potential of 
glioblastoma stem-like cells. Cell. 2014; 157: 580-594.

29. Zhang C, Moore LM, Li X, Yung WK, Zhang W. IDH1/2 mutations target a key hallmark of cancer by deregulating cellular metabolism in glioma. Neuro Oncol. 2013; 15: 1114-26.

30. Murata Y, Sun-Wada GH, Yoshimizu T, Yamamoto A, Wada Y, Futai M. Differential localization of the vacuolar $\mathrm{H}+$ pump with $\mathrm{G}$ subunit isoforms (G1 and G2) in mouse neurons. J Biol Chem. 2002; 277: 36296-36303.

31. Torigoe $\mathrm{T}$, Izumi $\mathrm{H}$, Ishiguchi $\mathrm{H}$, Uramoto $\mathrm{H}$, Murakami $\mathrm{T}$, Ise T, Yoshida Y, Tanabe M, Nomoto M, Itoh H, Kohno K. Enhanced expression of the human vacuolar $\mathrm{H}+$-ATPase $\mathrm{c}$ subunit gene (ATP6L) in response to anticancer agents. J Biol Chem. 2002; 277: 36534-36543.

32. Hendrix A, Sormunen R, Westbroek W, Lambein K, Denys H, Sys G, Braems G, Van den Broecke R, Cocquyt V, Gespach C, Bracke M, De Wever O. Vacuolar H+ ATPase expression and activity is required for Rab27B-dependent invasive growth and metastasis of breast cancer. Int $\mathrm{J}$ Cancer. 2013; 133: 843-854.

33. Hinton A, Sennoune SR, Bond S, Fang M, Reuveni M, Sahagian GG, Jay D, Martinez-Zaguilan R, Forgac M. Function of a subunit isoforms of the V-ATPase in $\mathrm{pH}$ homeostasis and in vitro invasion of MDA-MB231 human breast cancer cells. J Biol Chem. 2009; 284: 16400-16408.

34. Kubisch R, Fröhlich T, Arnold GJ, Schreiner L, von Schwarzenberg K, Roidl A, Vollmar AM, Wagner E. V-ATPase inhibition by archazolid leads to lysosomal dysfunction resulting in impaired cathepsin B activation in vivo. Int J Cancer. 2014; 134: 2478-2488.

35. Kobia F, Duchi S, Deflorian G, Vaccari T. Pharmacologic inhibition of vacuolar H+ ATPase reduces physiologic and oncogenic Notch signaling. Mol Oncol. 2014; 8: 207-220.

36. Warton K, Tonini R, Fairlie WD, Matthews JM, Valenzuela SM, Qiu MR, Wu WM, Pankhurst S, Bauskin AR, Harrop SJ, Campbell TJ, Curmi PM, Breit SN, et al. Recombinant CLIC1 (NCC27) assembles in lipid bilayers via a $\mathrm{pH}$ dependent two-state process to form chloride ion channels with identical characteristics to those observed in Chinese hamster ovary cells expressing CLIC1. J Biol Chem. 2002; 277: 26003-11.

37. Mokrý J, Cízková D, Filip S, Ehrmann J, Osterreicher J, Kolár Z, English D. Nestin expression by newly formed human blood vessels. Stem Cells Dev. 2004; 13: 658-664.

38. Alliot F, Rutin J, Leenen PJ, Pessac B. Pericytes and periendothelial cells of brain parenchyma vessels coexpress aminopeptidase N, aminopeptidase A, and nestin. J Neurosci Res. 1999; 58: 367-378.

39. Lomonaco SL, Finniss S, Xiang C, Decarvalho A, Umansky F, Kalkanis SN, Mikkelsen T, Brodie C. The induction of autophagy by gamma-radiation contributes to the radioresistance of glioma stem cells. Int J Cancer. 2009; 125: 717-722.

40. Del Gobbo A, Vaira V, Ferrari L, Patriarca C, Di Cristofori
A, Ricca D, Caroli M, Rampini P, Bosari S, and Ferrero S. The Oncofetal Protein IMP3: A Novel Grading Tool and Predictor of Poor Clinical Outcome in Human Gliomas. BioMed Research International, Article ID 413897, in press.

41. Louis D, Ohgaki H, Wiestler O, Cavenee W, Burger P, Jouvet A, Scheithauer B, Kleihues P. The 2007 WHO Classification of Tumours of the Central Nervous System. Acta Neuropathol. 2007; 114: 97-109.

42. Stupp R, Tonn J-C, Brada M, Pentheroudakis G. On behalf of the ESMO Guidelines Working Group. High-grade malignant glioma: ESMO Clinical Practice Guidelines for diagnosis, treatment and follow-up. Annals of Oncology. 2010; 21 (Supplement 5): v190-v193.

43. Savi F, Forno I, Faversani A, Luciani A, Caldiera S, Gatti S, Foa P, Ricca D, Bulfamante G, Vaira V, Bosari S. miR$296 /$ Scribble axis is deregulated in human breast cancer and miR-296 restoration reduces tumour growth in vivo. Clin Sci (Lond). 2014; 127: 233-242.

44. Ghosh JC, Siegelin MD, Vaira V, Faversani A, Tavecchio M, Chae YC, Lisanti S, Rampini P, Giroda M, Caino MC, Seo JH, Kossenkov AV, Michalek RD, et al. Adaptive mitochondrial reprogramming and resistance to $\mathrm{PI} 3 \mathrm{~K}$ therapy. J Natl Cancer Inst. 2015; 107. pii: dju502.

45. Vaira V, Faversani A, Martin NM, Garlick DS, Ferrero S, Nosotti M, Kissil JL, Bosari S, Altieri DC. Regulation of lung cancer metastasis by Klf4-Numb-like signaling. Cancer Res. 2013; 73: 2695-2705.

46. Caino MC, Chae YC, Vaira V, Ferrero S, Nosotti M, Martin NM, Weeraratna A, O'Connell M, Jernigan D, Fatatis A, Languino LR, Bosari S, Altieri DC. Metabolic stress regulates cytoskeletal dynamics and metastasis of cancer cells. J Clin Invest. 2013; 123: 2907-20. 